\title{
Spectral signatures of the BCS-BEC crossover in the excitonic insulator phase of the extended Falicov-Kimball model
}

\author{
Van-Nham Phan ${ }^{1}$, Klaus W. Becker ${ }^{2}$ and Holger Fehske ${ }^{1}$ \\ ${ }^{1}$ Institut für Physik, Ernst-Moritz-Arndt-Universität Greifswald, D-17487 Greifswald, Germany \\ ${ }^{2}$ Institut für Theoretische Physik, Technische Universität Dresden, D-01062 Dresden, Germany
}

(Dated: November 1, 2018)

\begin{abstract}
We explore the spontaneous formation of an excitonic insulator state at the semimetalsemiconductor transition of mixed-valence materials in the framework of the spinless Falicov-Kimball model with direct $f-f$ electron hopping. Adapting the projector-based renormalization method, we obtain a set of renormalization differential equations for the extended Falicov-Kimball model parameters and finally derive analytical expressions for the order parameter, as well as for the renormalized $c$ - and $f$-electron dispersions, momentum distributions, and wave-vector resolved single-particle spectral functions. Our numerical results proved the valence transition picture, related to the appearance of the excitonic insulator phase, in the case of overlapping $c$ and $f$ bands. Thereby the photoemission spectra show significant differences between the weak-to-intermediate and intermediateto-strong Coulomb attraction regimes, indicating a BCS-BEC transition of the excitonic condensate.
\end{abstract}

PACS numbers: 71.28.+d, 71.35.Lk, 71.30.+h

\section{INTRODUCTION}

The idea that an excitonic phase appears - under certain circumstances - at the semiconductor-semimetal

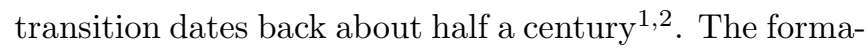
tion of excitons is driven by the Coulomb attraction between conduction-band electrons and valence-band holes. Provided a large enough number of sufficiently long-lived excitons was created, a subsequent spontaneous condensation of these composite Bose quasiparticles may set in. The excitonic instability is expected to happen, when semimetals with very small band overlap or semiconductors with very small band gap are cooled to extremely low temperatures ${ }^{3,4}$. The excitonic condensate typifies a macroscopic phase-coherent, insulating state, which separates the semimetal from the semiconductor (see Fig. (1).

Surprisingly, to date, there is no free of doubt realization of the excitonic insulator (EI) state in nature. Nowadays experiments report data, however, which strongly support the theoretical predictions of the EI phase. Along this line, experiments on coupled quantumwell structures, e.g., have shown unusual properties which were inferred as indications of excitonic condensation $^{5}$. Temperature dependent angle-resolved photoelectron spectroscopy (ARPES) on $1 T-\mathrm{TiSe}_{2}$ transitionmetal dichalcogenides are in favor of the EI scenario as driving force for the observed charge-density-wave transition ${ }^{6}$. X-ray photoemission spectroscopy and ARPES on quasi one-dimensional (1D) $\mathrm{Ta}_{2} \mathrm{NiSe}_{5}$ reveal that the ground state can be viewed as EI state between the Ni $3 d-$ Se $4 p$ hole and the Ta $5 d$ electron ${ }^{7}$. Further real-system candidates for the EI state are pressuresensitive rare-earth chalcogenides, such as mixed-valence $\mathrm{TmSe}_{0.45} \mathrm{Te}_{0.55}$. For this compounds electrical and thermal (transport) measurements indicate exciton condensation, at temperatures below $20 \mathrm{~K}$ in the pressure range between 5 and $11 \mathrm{kbar}^{8}$.

Also from the theoretical side the existence of the EI is still controversial. Most of the early mean-field approaches work with an effective-mass Mott-Wannier-type exciton model and exploit the analogy to the BCS theory of superconductivity $\stackrel{10}{\underline{1}}$ (for a more recent calculation of the phase diagram see Refs. 9, 11). Here the major problem is that the excitonic phases (excitonic gas, EI) turn out to be unstable against a metallic electron-hole liq-

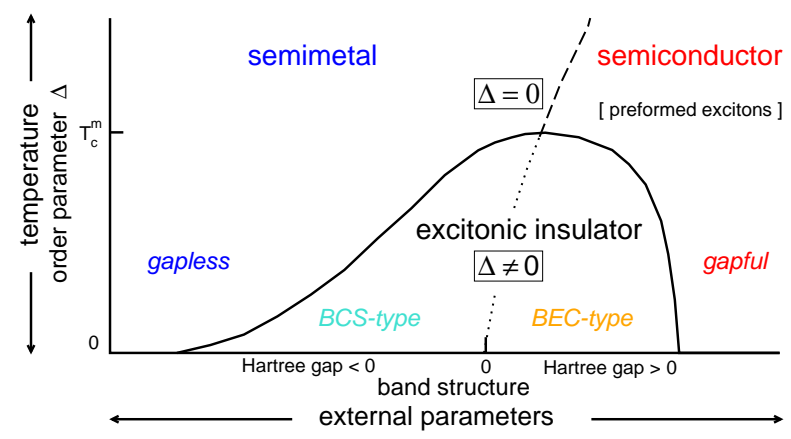

FIG. 1: (Color online) EI formation and BCS-BEC transition scenario. At the semimetal-semiconductor transition the ground state of the system may become unstable with respect to the spontaneous formation of excitons near the point at which band overlap occurs. Starting from a semimetal with small density of electrons and holes (such that the Coulomb interaction is basically unscreened), the number of free carriers varies discontinuously under an applied perturbation, signaling a phase transition ${ }^{1}$. Approaching the transition from the semiconductor side, an anomaly occurs when the band gap, tuned, e.g., by external pressure, becomes less than the exciton binding energy ${ }^{2}$. Depending on from which side of the semimetal-semiconductor transition the EI is reached, the EI can be viewed either as BCS condensate of loosely-bound electron-hole pairs or as BEC of preformed tightly-bound excitons ${ }^{9}$. A finite order parameter $\Delta$ indicates the new distorted phase of the crystal, with coherence between conduction- and valence-band electrons and a gap for charge excitations. 
uid $^{12,13}$. At present, Falicov-Kimball-type models seem to be the most promising candidates for realizing collective exciton phases. This particularly holds for the extended Falicov-Kimball model (EFKM), which includes a direct $f$ - $f$ electron hopping term, that-having again the $\operatorname{Tm}[\mathrm{Se}, \mathrm{Te}]$ system in mind - is certainly more realistic than entirely localized $f$ electrons. By means of unbiased constrained path Monte Carlo (CPMC) simulations the EFKM has been proven to exhibit critical excitonic correlations (an EI ground state) in case of 1D $(2 \mathrm{D})^{\underline{14,15}}$. Subsequent Hartree-Fock calculations yield the ground-state phase diagram of the 2D EFKM in excellent agreement with the CPMC data ${ }^{16}$, supporting the applicability of such mean-field approaches also in the 3D situation 16,17 . For the 3D EFKM, the existence of the EI phase was corroborated by more sophisticated slave-boson approaches ${ }^{18,19}$.

Assuming that $f$-c electron coherence may lead to an EI phase in the EFKM, the properties of the excitonic state should be explored in more detail. In this regard, the anticipated 'BCS-BEC crossover' scenario ${ }^{9,11,20}$, connecting the physics of BCS superconductivity with that of Bose-Einstein condensates (BECs), is of vital importance. Calculating the Frenkel-type exciton propagator within a random phase approximation scheme, the existence of excitonic bound-states has been established for the EFKM also above $T_{c}$, on the semiconductor side of the semiconductor-semimetal transition 21 . No boundstates were found on the semi-metallic side. Accordingly the condensation process should differ by its nature: While 'exciton' formation and condensation simultaneously take place on the semi-metallic (BCS) side, preformed excitons will condense on the semiconducting (BEC) side as the temperature is lowered (cf. Fig. 1). Looking at the EI order parameter $\Delta$ only, it seems difficult to examine the BCS-BEC crossover. The gap equation for $\Delta$ will of course not discriminate between both regimes. Photoemission spectroscopy, on the other hand, will directly probe the elementary excitations and energy dispersion and therefore provides extremely useful information about the BCS-BEC crossover. This has been shown quite recently in the context of ultracold (atomic) Fermi gases 22 .

In this work, we will follow this perspective and examine the EI phase in terms of the EFKM particularly with regard to a BCS-BEC crossover. To this end, we analyze the equilibrium and spectral properties of the model at zero temperature, using the so-called projective renormalization method $(\mathrm{PRM})^{23,24}$. This technique has already been successively applied to a great variety of many-body problems 25,26 . Here we calculate and discuss the photoemission spectra of the EFKM in order to probe the signatures of the excitonic condensate. The paper is organized as follows. Section II introduces the EFKM. The theoretical approach is outlined in Sec. III, where the general concept of the PRM is resumed in Sec. III. A and explicit expressions for the renormalization differential equations, particle number expectation values, cor- relation functions and single-particle spectral functions are given in Sec. III. B. Section IV presents the corresponding numerical results. Our main conclusions can be found in Sec. V.

\section{EXTENDED FALICOV-KIMBALL MODEL}

The Hamiltonian for the EFKM is written

$$
\mathcal{H}=\sum_{\mathbf{k}} \bar{\varepsilon}_{\mathbf{k}}^{c} c_{\mathbf{k}}^{\dagger} c_{\mathbf{k}}+\sum_{\mathbf{k}} \bar{\varepsilon}_{\mathbf{k}}^{f} f_{\mathbf{k}}^{\dagger} f_{\mathbf{k}}+\sum_{i} U n_{i}^{c} n_{i}^{f},
$$

where $c_{\mathbf{k}}^{\dagger}\left(c_{\mathbf{k}}\right)$ and $f_{\mathbf{k}}^{\dagger}\left(f_{\mathbf{k}}\right)$ are the creation (annihilation) operators in momentum (k-) space of spinless $c$ and $f$-electrons, respectively, and $n_{i}^{c}$ and $n_{i}^{f}$ are the corresponding occupation numbers in real space. The Fourier-transformed fermionic operators are defined via $\eta_{\mathbf{k}}^{\dagger}=\frac{1}{\sqrt{N}} \sum_{i} \eta_{i}^{\dagger} e^{i \mathbf{k R}_{i}}$, where $\eta=c, f$, and the $\eta$-fermion dispersion is

$$
\bar{\varepsilon}_{\mathbf{k}}^{\eta}=\varepsilon^{\eta}-t^{\eta} \gamma_{\mathbf{k}}-\mu
$$

with on-site energy $\varepsilon^{\eta}$. In Eq. (2), $\mu$ denotes the chemical potential. In the tight-binding limit, on a $\mathrm{D}$-dimensional hypercubic lattice, we have $\gamma_{\mathbf{k}}=2 \sum_{d=1}^{D} \cos k_{d}$. The sign of $t^{c} t^{f}$ determines whether we deal with a direct $\left(t^{c} t^{f}<0\right)$ or indirect $\left(t^{c} t^{f}>0\right)$ band-gap situation. Usually, the $c$-electrons are considered to be 'light' and their hopping integral is taken to be the unit of energy $\left(t^{c}=1\right)$, while the $f$-electrons are 'heavy', i.e., $\left|t^{f}\right|<1$. For $t^{f} \equiv 0$ (dispersionless $f$ band), the local $f$-electron number is strictly conserved 17 . The third term in the Hamiltonian (11) represents the Coulomb interaction between $c$ and $f$ electrons at the same lattice site. Hence, if the $c$ and $f$ bands are degenerate, $\varepsilon^{c}=\varepsilon^{f}$ and $t^{c}=t^{f}$, the EFKM reduces to the standard Hubbard model.

In order to address the formation of the EI state in the EFKM, we look for a non-vanishing excitonic expectation value $\left\langle c^{\dagger} f\right\rangle$, indicating a kind of spontaneous symmetry breaking due the pairing of $c$ electrons $\left(t^{c}>0\right)$ with $f$ holes $\left(t^{f}<0\right)$. This is quite similar to the problem of electronic ferroelectricity, where $\left\langle c^{\dagger} f\right\rangle \neq 0$ causes electrical polarizability without an interband transition driving field, provided the $c$ and $f$ states have different parity ${ }^{15.17}$. Thereby, depending on the sign of $t_{f}$ (direct or indirect gap), ferro- or antiferroelectric phases may exist.

To proceed, we introduce two-particle interaction operators in momentum space,

$$
a_{\mathbf{k}_{1} \mathbf{k}_{2} \mathbf{k}_{3}}=c_{\mathbf{k}_{1}}^{\dagger} c_{\mathbf{k}_{2}} f_{\mathbf{k}_{3}}^{\dagger} f_{\mathbf{k}_{1}+\mathbf{k}_{3}-\mathbf{k}_{2}},
$$

and rewrite the EFKM Hamiltonian (10) in a normalordered form ${ }^{27}$ :

$$
\begin{aligned}
\mathcal{H} & =\sum_{\mathbf{k}} \varepsilon_{\mathbf{k}}^{c}: c_{\mathbf{k}}^{\dagger} c_{\mathbf{k}}:+\sum_{\mathbf{k}} \varepsilon_{\mathbf{k}}^{f}: f_{\mathbf{k}}^{\dagger} f_{\mathbf{k}}: \\
& -\sum_{\mathbf{k}}\left(\Delta: f_{\mathbf{k}}^{\dagger} c_{\mathbf{k}}:+ \text { H.c. }\right)+\frac{U}{N} \sum_{\mathbf{k}_{1} \mathbf{k}_{2} \mathbf{k}_{3}}: a_{\mathbf{k}_{1} \mathbf{k}_{2} \mathbf{k}_{3}}:
\end{aligned}
$$


where

$$
\Delta=\frac{U}{N} \sum_{\mathbf{k}} d_{\mathbf{k}}
$$

with $d_{\mathbf{k}}=\left\langle c_{\mathbf{k}}^{\dagger} f_{\mathbf{k}}\right\rangle$, plays the role of the EI order parameter. Note that choosing the normal-ordered representation of operators, the symmetry of the Hamiltonian is explicitly broken, and iterating the self-consistency equation derived below will readily give (meta-) stable solutions $^{28}$. In the Hamiltonian (4), the on-site energies were shifted by a Hartree term,

$$
\varepsilon_{\mathbf{k}}^{c(f)}=\bar{\varepsilon}_{\mathbf{k}}^{c(f)}+U\left\langle n^{f(c)}\right\rangle,
$$

where $\left\langle n^{\eta}\right\rangle=\frac{1}{N} \sum_{\mathbf{k}}\left\langle\eta_{\mathbf{k}}^{\dagger} \eta_{\mathbf{k}}\right\rangle$ are the particle number densities of $c$ or $f$ electrons for a system with $N$ lattice sites. In what follows, we consider the half-filled band case, i.e., we fix the total electron density $n=\left\langle n^{c}\right\rangle+\left\langle n^{f}\right\rangle=1$.

\section{THEORETICAL APPROACH}

\section{A. Projector-based renormalization method}

The PRM was recently developed with the aim to diagonalize many-particle systems ${ }^{29}$. One of the main advantages of the method is to find broken symmetry solutions of phase transitions ${ }^{30}$. For example, in Ref. 26 the method was successfully applied to the $t-J$ model in order to study superconducting $d$-wave solutions for cuprates.

The PRM starts from the decomposition of a given many-particle Hamiltonian into an 'unperturbed' part $\mathcal{H}_{0}$ and into a 'perturbation' $\mathcal{H}_{1}$, where the unperturbed part $\mathcal{H}_{0}$ should be solvable. Suppose all diagonal matrix elements of $\mathcal{H}_{1}$ between eigenvectors of $\mathcal{H}_{0}$ vanish, the part $\mathcal{H}_{1}$ accounts for all transitions between the eigenstates of $\mathcal{H}_{0}$ with nonzero transition energies. Then, the first goal of the PRM is to transform the initial Hamiltonian into an effective Hamiltonian $\mathcal{H}_{\lambda}$ which contains no longer transition operators with energies larger than some chosen cutoff $\lambda$. Thereby, the Hamiltonian $\mathcal{H}_{\lambda}$ is formally obtained by applying a unitary transformation

$$
\mathcal{H}_{\lambda}=e^{\mathcal{X}_{\lambda}} \mathcal{H} e^{-\mathcal{X}_{\lambda}} \text {. }
$$

The transformed Hamiltonian $\mathcal{H}_{\lambda}$, which has the same eigenspectrum as the original Hamiltonian $\mathcal{H}$, can again be decomposed into two parts

$$
\mathcal{H}_{\lambda}=\mathcal{H}_{0, \lambda}+\mathcal{H}_{1, \lambda}
$$

Due to construction, all matrix elements $\left\langle n_{\lambda}\left|\mathcal{H}_{1, \lambda}\right| m_{\lambda}\right\rangle$ of $\mathcal{H}_{1, \lambda}$ with energy differences $\left|E_{n}^{\lambda}-E_{m}^{\lambda}\right|>\lambda$ should vanish, i.e. $\left\langle n_{\lambda}\left|\mathcal{H}_{1, \lambda}\right| m_{\lambda}\right\rangle=0$, where $E_{n}^{\lambda}$ and $\left|n_{\lambda}\right\rangle$ are the new renormalized eigenvalues and eigenstates of $\mathcal{H}_{0, \lambda}$. Note that neither $\left|n_{\lambda}\right\rangle$ nor $\left|m_{\lambda}\right\rangle$ have to be low-energy eigenstates of $\mathcal{H}_{0, \lambda}$. To ensure hermiticity of $\mathcal{H}_{\lambda}$, the generator $\mathcal{X}_{\lambda}$ of the unitary transformation has to satisfy $\mathcal{X}_{\lambda}^{\dagger}=-\mathcal{X}_{\lambda}$.

A crucial idea for the elimination procedure in the PRM is to introduce generalized projection operators $\mathbf{P}_{\lambda}$ and $\mathbf{Q}_{\lambda}=\mathbf{1}-\mathbf{P}_{\lambda}$. Here $\mathbf{P}_{\lambda}$ is defined by

$$
\mathbf{P}_{\lambda} \mathcal{A}=\sum_{\substack{m, n \\\left|E_{n}^{\lambda}-E_{m}^{\lambda}\right| \leq \lambda}}\left|n_{\lambda}\right\rangle\left\langle m_{\lambda}\right|\left\langle n_{\lambda}|\mathcal{A}| m_{\lambda}\right\rangle,
$$

applied on any operator variable $\mathcal{A}$ of the Hilbert space of the system. Note that in expression (9) only states $\left|n_{\lambda}\right\rangle$ and $\left|m_{\lambda}\right\rangle$ satisfying $\left|E_{n}^{\lambda}-E_{m}^{\lambda}\right| \leq \lambda$ contribute to the transition matrix. Thus, $\mathbf{P}_{\lambda}$ projects on the low energy transitions of $\mathcal{A}$, whereas the orthogonal complement $\mathbf{Q}_{\lambda}$ projects on the high-energy transitions of $\mathcal{A}$. To find an appropriate generator $\mathcal{X}_{\lambda}$ for the unitary transformation from $\mathcal{H}$ to $\mathcal{H}_{\lambda}$, the obvious relation

$$
\mathbf{Q}_{\lambda} \mathcal{H}_{\lambda}=0
$$

has to be fulfilled.

In the original version of the $\mathrm{PRM}^{23}$, the elimination procedure is performed step-wise. Suppose $\Lambda$ is the largest transition energy of the original Hamiltonian $\mathcal{H}$, in the first elimination step all transitions in an energy shell of width $\Delta \lambda$ between $\Lambda$ and $\Lambda-\Delta \lambda$ will be removed. The subsequent steps remove, roughly speaking, all transitions in the next shell of width $\Delta \lambda$ between $\Lambda-\Delta \lambda$ and $\Lambda-2 \Delta \lambda$, and so on. The unitary transformation for the intermediate step from a cutoff $\lambda$ to the new cutoff $\lambda-\Delta \lambda$ reads

$$
\mathcal{H}_{\lambda-\Delta \lambda}=e^{\mathcal{X}_{\lambda, \Delta \lambda}} \mathcal{H}_{\lambda} e^{-\mathcal{X}_{\lambda, \Delta \lambda}}
$$

Here, the generator $\mathcal{X}_{\lambda, \Delta \lambda}$ has to fulfill the requirement

$$
\mathbf{Q}_{\lambda-\Delta \lambda} \mathcal{H}_{\lambda-\Delta \lambda}=0
$$

in analogy to (10). Thus $\mathcal{H}_{\lambda-\Delta \lambda}$ has no matrix elements that connect eigenstates of $\mathcal{H}_{0, \lambda-\Delta \lambda}$ with energy differences larger than $\lambda-\Delta \lambda$.

By help of Eqs. (11) and (12), the generator $\mathcal{X}_{\lambda, \Delta \lambda}$ can easily be constructed in perturbation theory with respect to $\mathcal{H}_{1, \lambda}$. Up to first order it reads

$$
\mathcal{X}_{\lambda, \Delta \lambda}=\frac{1}{\mathbf{L}_{0, \lambda}} \mathbf{Q}_{\lambda-\Delta \lambda} \mathcal{H}_{1, \lambda}=\mathbf{Q}_{\lambda-\Delta \lambda} \mathcal{X}_{\lambda, \Delta \lambda}
$$

Here, $\mathbf{L}_{0, \lambda}$ is the Liouville superoperator of the unperturbed Hamiltonian $\mathcal{H}_{0, \lambda}$, which is defined by the commutator of $\mathcal{H}_{0, \lambda}$ with any operator $\mathcal{A}$ on which $\mathbf{L}_{0, \lambda}$ is applied, i.e. $\mathbf{L}_{0, \lambda} \mathcal{A}=\left[\mathcal{H}_{0, \lambda}, \mathcal{A}\right]$. Note, however, that the generator $\mathcal{X}_{\lambda, \Delta \lambda}$ is not completely fixed by Eqs. (11) and (12). In fact, only the part $\mathbf{Q}_{\lambda-\Delta \lambda} \mathcal{X}_{\lambda, \Delta \lambda}$ is determined by equation (12). The part $\mathbf{P}_{\lambda-\Delta \lambda} \mathcal{X}_{\lambda, \Delta \lambda}$ with only low-energy transitions can still be chosen arbitrarily and was set identical to zero in Eq. (13). Any physical quantities, which is evaluated in the framework of the PRM, is independent of a particular choice of $\mathbf{P}_{\lambda-\Delta \lambda} \mathcal{X}_{\lambda, \Delta \lambda} 24$. 
This freedom can be used to derive a continuous version of the method. Thereby, the low excitation part $\mathbf{P}_{\lambda-\Delta \lambda} \mathcal{X}_{\lambda, \Delta \lambda}$ is chosen proportional to $\Delta \lambda$, which allows to derive differential equations for the $\lambda$-dependence of the parameter values in the Hamiltonian during the renormalization procedure. As in the discrete version 23 , also in the continuous version the elimination starts from the original model and proceeds until $\lambda=0$. At this point, all transitions operators from $\mathcal{H}_{1}$ have been used up and the final Hamiltonian is diagonal or at least quasidiagonal which allows to evaluate expectation values. Note that the parameters of the renormalized Hamiltonian depend on the parameter values of the original model $\mathcal{H}$.

To evaluate expectation values of operators $\mathcal{A}$, formed with the full Hamiltonian, we have to apply the unitary transformation to $\mathcal{A}$ as well,

$$
\langle\mathcal{A}\rangle=\frac{\operatorname{Tr} \mathcal{A} e^{-\beta \mathcal{H}}}{\operatorname{Tr} e^{-\beta \mathcal{H}}}=\langle\mathcal{A}(\lambda)\rangle_{\mathcal{H}_{\lambda}}=\langle\tilde{\mathcal{A}}\rangle_{\tilde{\mathcal{H}}}
$$

where we define $\mathcal{A}(\lambda)=e^{\mathcal{X}_{\lambda}} \mathcal{A} e^{-\mathcal{X}_{\lambda}}, \tilde{\mathcal{A}}=\mathcal{A}(\lambda \rightarrow 0)$, and $\tilde{\mathcal{H}}=\mathcal{H}_{\lambda \rightarrow 0}$. Thus additional renormalization equations are required for $\mathcal{A}(\lambda)$.

\section{B. Application to the EFKM}

\section{Renormalization equations}

In order to derive the renormalization equations for the parameters of the Hamiltonian, we first decompose the original Hamiltonian $\mathcal{H}$ into two parts

$$
\mathcal{H}=\mathcal{H}_{0}+\mathcal{H}_{1}
$$

where

$$
\begin{aligned}
\mathcal{H}_{0} & =\sum_{\mathbf{k}} \varepsilon_{\mathbf{k}}^{c}: c_{\mathbf{k}}^{\dagger} c_{\mathbf{k}}:+\sum_{\mathbf{k}} \varepsilon_{\mathbf{k}}^{f}: f_{\mathbf{k}}^{\dagger} f_{\mathbf{k}}: \\
& +\sum_{\mathbf{k}}\left(\Delta: f_{\mathbf{k}}^{\dagger} c_{\mathbf{k}}:+ \text { H.c. }\right)
\end{aligned}
$$

and

$$
\mathcal{H}_{1}=\frac{U}{N} \sum_{\mathbf{k}_{1} \mathbf{k}_{2} \mathbf{k}_{3}}: a_{\mathbf{k}_{1} \mathbf{k}_{2} \mathbf{k}_{3}}:
$$

Note again that the perturbation $\mathcal{H}_{1}$ only contains the fluctuating operator part of the Coulomb repulsion $\propto$ $U$. Following the ideas of the PRM approach, we make the following ansatz for the renormalized Hamiltonian $\mathcal{H}_{\lambda}$ after all transitions with energies larger than $\lambda$ are integrated out:

$$
\mathcal{H}_{\lambda}=\mathcal{H}_{0, \lambda}+\mathcal{H}_{1, \lambda}
$$

with

$$
\begin{aligned}
\mathcal{H}_{0, \lambda}= & \sum_{\mathbf{k}} \varepsilon_{\mathbf{k}, \lambda}^{c}: c_{\mathbf{k}}^{\dagger} c_{\mathbf{k}}:+\sum_{\mathbf{k}} \varepsilon_{\mathbf{k}, \lambda}^{f}: f_{\mathbf{k}}^{\dagger} f_{\mathbf{k}}:+E_{\lambda} \\
& +\sum_{\mathbf{k}}\left(\Delta_{\mathbf{k}, \lambda}: f_{\mathbf{k}}^{\dagger} c_{\mathbf{k}}:+ \text { H.c. }\right), \\
\mathcal{H}_{1, \lambda}= & \frac{1}{N} \mathbf{P}_{\lambda} \sum_{\mathbf{k}_{1} \mathbf{k}_{2} \mathbf{k}_{3}} U_{\mathbf{k}_{1} \mathbf{k}_{2} \mathbf{k}_{3}, \lambda}: a_{\mathbf{k}_{1} \mathbf{k}_{2} \mathbf{k}_{3}}:
\end{aligned}
$$

Here, $\mathbf{P}_{\lambda}$ projects on all low-energy transitions with respect to the unperturbed Hamiltonian $\mathcal{H}_{0, \lambda}$ which are smaller than $\lambda$. Due to renormalization all prefactors in Eqs. (19), (20) may now depend on the wave vector $\mathbf{k}$ and on the energy cutoff $\lambda$. The quantity $E_{\lambda}$ is an energy shift which enters during the renormalization procedure. In order to evaluate the action of the superoperator $\mathbf{P}_{\lambda}$ on the interaction operator in $\mathcal{H}_{1, \lambda}$ one has to decompose the fluctuation operators : $a_{\mathbf{k}_{1} \mathbf{k}_{2} \mathbf{k}_{3}}$ : into eigenmodes of $\mathcal{H}_{0, \lambda}$. Obviously, the diagonalization of $\mathcal{H}_{0, \lambda}$ requires an additional unitary transformation. However, for the values of $U$, used in the numerical evaluation below, the mixing parameter $\Delta_{\mathbf{k}, \lambda}$ in Eq. (19) turns out to be always small compared to the energy difference $\left|\varepsilon_{\mathbf{k}, \lambda}^{c}-\varepsilon_{\mathbf{k}, \lambda}^{f}\right|$. This follows from the Hartree shifts of the one-particle energies in Eq. (6). Thus, using as approximation $\mathbf{L}_{0, \lambda} c_{\mathbf{k}}^{\dagger}=\varepsilon_{\mathbf{k}}^{c} c_{\mathbf{k}}^{\dagger}$ and $\mathbf{L}_{0, \lambda} f_{\mathbf{k}}^{\dagger}=\varepsilon_{\mathbf{k}}^{f} f_{\mathbf{k}}^{\dagger}$, we can conclude

$\mathcal{H}_{1, \lambda}=\frac{1}{N} \sum_{\mathbf{k}_{1} \mathbf{k}_{2} \mathbf{k}_{3}} \Theta\left(\lambda-\left|\tilde{\eta}_{\mathbf{k}_{1} \mathbf{k}_{2} \mathbf{k}_{3}, \lambda}\right|\right) U_{\mathbf{k}_{1} \mathbf{k}_{2} \mathbf{k}_{3}, \lambda}: a_{\mathbf{k}_{1} \mathbf{k}_{2} \mathbf{k}_{3}}:$,

where

$$
\tilde{\eta}_{\mathbf{k}_{1} \mathbf{k}_{2} \mathbf{k}_{3}, \lambda}=\varepsilon_{\mathbf{k}_{1}, \lambda}^{c}-\varepsilon_{\mathbf{k}_{2}, \lambda}^{c}+\varepsilon_{\mathbf{k}_{3}, \lambda}^{f}-\varepsilon_{\mathbf{k}_{1}+\mathbf{k}_{3}-\mathbf{k}_{2}, \lambda}^{f}
$$

is the approximate excitation energy of $: a_{\mathbf{k}_{1} \mathbf{k}_{2} \mathbf{k}_{3}}$, , i.e.

$$
\mathbf{L}_{0, \lambda}: a_{\mathbf{k}_{1} \mathbf{k}_{2} \mathbf{k}_{3}}:=\tilde{\eta}_{\mathbf{k}_{1} \mathbf{k}_{2} \mathbf{k}_{3}, \lambda}: a_{\mathbf{k}_{1} \mathbf{k}_{2} \mathbf{k}_{3}}: .
$$

The $\Theta$-function in Eq. (21) ensures that only transitions with excitation energies smaller than $\lambda$ remain in $\mathcal{H}_{1, \lambda}$.

By integrating out all transitions between the cutoff $\Lambda$ of the original model and $\lambda=0$, all parameters of the original model will become renormalized. To find their $\lambda$ dependence, we derive renormalization equations for the parameters $\varepsilon_{\mathbf{k}, \lambda}^{c}, \varepsilon_{\mathbf{k}, \lambda}^{f}, \Delta_{\mathbf{k}, \lambda}$, and $U_{\mathbf{k}_{1} \mathbf{k}_{2} \mathbf{k}_{3}, \lambda}$. The initial parameter values are determined by the original model $(\lambda=\Lambda)$ :

$$
\begin{array}{ll}
\varepsilon_{\mathbf{k}, \Lambda}^{c}=\varepsilon_{\mathbf{k}}^{c}, & \Delta_{\mathbf{k}, \Lambda}=\Delta, \\
\varepsilon_{\mathbf{k}, \Lambda}^{f}=\varepsilon_{\mathbf{k}}^{f}, & U_{\mathbf{k}_{1} \mathbf{k}_{2} \mathbf{k}_{3}, \Lambda}=U .
\end{array}
$$

Note that the energy shift $E_{\lambda}$ in $\mathcal{H}_{0, \lambda}$ has no effect on expectation values and will be left out in what follows.

Next we have to construct the generator $\mathcal{X}_{\lambda, \Delta \lambda}$ of transformation (11). Using relation (23), the high transition energy part reads in lowest order perturbation the- 
ory according to Eqs. (13)

$$
\begin{array}{r}
\mathbf{Q}_{\lambda-\Delta \lambda} \mathcal{X}_{\lambda, \Delta \lambda}=\frac{1}{N} \sum_{\mathbf{k}_{1} \mathbf{k}_{2} \mathbf{k}_{3}} \frac{U_{\mathbf{k}_{1} \mathbf{k}_{2} \mathbf{k}_{3}, \lambda}}{\tilde{\eta}_{\mathbf{k}_{1} \mathbf{k}_{2} \mathbf{k}_{3}, \lambda}}\left(1-\Theta_{\mathbf{k}_{1} \mathbf{k}_{2} \mathbf{k}_{3}, \lambda-\Delta \lambda}\right) \\
\times \Theta_{\mathbf{k}_{1} \mathbf{k}_{2} \mathbf{k}_{3}, \lambda}: a_{\mathbf{k}_{1} \mathbf{k}_{2} \mathbf{k}_{3}}:, \quad(26)
\end{array}
$$

where we have defined $\Theta_{\mathbf{k}_{1} \mathbf{k}_{2} \mathbf{k}_{3}, \lambda}=\Theta\left(\lambda-\left|\tilde{\eta}_{\mathbf{k}_{1} \mathbf{k}_{2} \mathbf{k}_{3}, \lambda}\right|\right)$. Here the product of the two $\Theta$-function assures that only excitations between $\lambda-\Delta \lambda$ and $\lambda$ are eliminated by the unitary transformation (11). As mentioned before, in the present approach we prefer to use a continuous version of the PRM approach which is based on the choice of the orthogonal complement part $\mathbf{P}_{\lambda-\Delta \lambda} \mathcal{X}_{\lambda, \Delta \lambda}$ of the generator. Thereby, $\mathbf{P}_{\lambda-\Delta \lambda} \mathcal{X}_{\lambda, \Delta \lambda}$ is chosen proportional to $\Delta \lambda$, which means that $\mathbf{Q}_{\lambda-\Delta \lambda} \mathcal{X}_{\lambda, \Delta \lambda}$ can be neglected in the limit $\Delta \lambda \rightarrow 0 \underline{24}$. With $\mathcal{X}_{\lambda, \Delta \lambda} \approx \mathbf{P}_{\lambda-\Delta \lambda} \mathcal{X}_{\lambda, \Delta \lambda}$, the following operator form for the generator can be used

$$
\begin{array}{r}
\mathcal{X}_{\lambda, \Delta \lambda}=\frac{\Delta \lambda}{N} \sum_{\mathbf{k}_{1} \mathbf{k}_{2} \mathbf{k}_{3}} \tilde{\alpha}_{\mathbf{k}_{1} \mathbf{k}_{2} \mathbf{k}_{3}, \lambda} \Theta_{\mathbf{k}_{1} \mathbf{k}_{2} \mathbf{k}_{3}, \lambda-\Delta \lambda} \\
\times \Theta_{\mathbf{k}_{1} \mathbf{k}_{2} \mathbf{k}_{3}, \lambda}: a_{\mathbf{k}_{1} \mathbf{k}_{2} \mathbf{k}_{3}}:,
\end{array}
$$

where the operators are taken over from expression (26). Note that the two $\Theta$-functions guarantee that expression (27) corresponds to the generator part with low energy excitations only. For the coefficients $\tilde{\alpha}_{\mathbf{k}_{1} \mathbf{k}_{2} \mathbf{k}_{3}, \lambda}$ we make the following ansatz:

$$
\tilde{\alpha}_{\mathbf{k}_{1} \mathbf{k}_{2} \mathbf{k}_{3}, \lambda}=\frac{\tilde{\eta}_{\mathbf{k}_{1} \mathbf{k}_{2} \mathbf{k}_{3}, \lambda}}{\kappa\left(\lambda-\left|\tilde{\eta}_{\mathbf{k}_{1} \mathbf{k}_{2} \mathbf{k}_{3}, \lambda}\right|\right)^{2}} U_{\mathbf{k}_{1} \mathbf{k}_{2} \mathbf{k}_{3}, \lambda}
$$

which is an appropriate choice in the continuous version of the $\mathrm{PRM}^{24}$. The constant $\kappa$ in (28) denotes an energy constant to ensure that the parameter $\tilde{\alpha}_{\mathbf{k}_{1} \mathbf{k}_{2} \mathbf{k}_{3}, \lambda}$ has the correct dimension of an inverse energy. At first glance, one might expect that $\tilde{\alpha}_{\mathbf{k}_{1} \mathbf{k}_{2} \mathbf{k}_{3}, \lambda}$ diverges at $\lambda=\left|\tilde{\eta}_{\mathbf{k}_{1} \mathbf{k}_{2} \mathbf{k}_{3}, \lambda}\right|$. Instead it vanishes exponentially at this point which follows from the renormalization equation for $U_{\mathbf{k}_{1} \mathbf{k}_{2} \mathbf{k}_{3}, \lambda}$, given below.

Our aim is to derive renormalization equations for renormalized Hamiltonian. The transformation (11) relates the Hamiltonian $\mathcal{H}_{\lambda}$ at cutoff $\lambda$ to that at the reduced cutoff $\lambda-\Delta \lambda$. With Eq. (27) one finds in the limit $\Delta \lambda \rightarrow 0$ :

$$
\frac{d \mathcal{H}_{\lambda}}{d \lambda}=-\frac{1}{N} \sum_{\mathbf{k}_{1} \mathbf{k}_{2} \mathbf{k}_{3}} \tilde{\alpha}_{\mathbf{k}_{1} \mathbf{k}_{2} \mathbf{k}_{3}, \lambda} \Theta_{\mathbf{k}_{1} \mathbf{k}_{2} \mathbf{k}_{3}, \lambda}\left[: a_{\mathbf{k}_{1} \mathbf{k}_{2} \mathbf{k}_{3}}:, \mathcal{H}_{\lambda}\right] .
$$

Note that the evaluation of the commutator also leads to new operators which are not present in the ansatz (18) for $\mathcal{H}_{\lambda}$. Therefore an additional factorization has to be used. Comparison with the generic derivation of (18) leads to the following set of coupled renormalization equations which describe the $\lambda$-dependent renormalization of the parameters of $\mathcal{H}_{\lambda}$ :

$$
\begin{aligned}
\frac{d \varepsilon_{\mathbf{k}, \lambda}^{c}}{d \lambda}=- & \frac{1}{N^{2}} \sum_{\mathbf{k}_{1} \mathbf{k}_{2}} U_{\mathbf{k}_{1} \mathbf{k} \mathbf{k}_{2}, \lambda} \tilde{\alpha}_{\mathbf{k k}_{1}, \mathbf{k}_{1}+\mathbf{k}_{2}-\mathbf{k}, \lambda}\left(1-\left\langle n_{\mathbf{k}_{1}}^{c}\right\rangle\right) \\
& \times\left(\left\langle n_{\mathbf{k}_{1}+\mathbf{k}_{2}-\mathbf{k}}^{f}\right\rangle-\left\langle n_{\mathbf{k}_{2}}^{f}\right\rangle\right) \\
- & \frac{1}{N^{2}} \sum_{\mathbf{k}_{1} \mathbf{k}_{2}} U_{\mathbf{k}_{1} \mathbf{k}_{2}, \lambda} \tilde{\alpha}_{\mathbf{k}_{1} \mathbf{k}, \mathbf{k}+\mathbf{k}_{2}-\mathbf{k}_{1}, \lambda}\left\langle n_{\mathbf{k}_{1}}^{c}\right\rangle \\
& \times\left(\left\langle n_{\mathbf{k}+\mathbf{k}_{2}-\mathbf{k}_{1}}^{f}\right\rangle-\left\langle n_{\mathbf{k}_{2}}^{f}\right\rangle\right), \\
\frac{d \varepsilon_{\mathbf{k}, \lambda}^{f}}{d \lambda}=- & \frac{1}{N^{2}} \sum_{\mathbf{k}_{1} \mathbf{k}_{2}} U_{\mathbf{k}_{1} \mathbf{k}_{2}, \mathbf{k}-\mathbf{k}_{1}+\mathbf{k}_{2}, \lambda} \tilde{\alpha}_{\mathbf{k}_{2} \mathbf{k}_{1} \mathbf{k}, \lambda}\left\langle n_{\mathbf{k}-\mathbf{k}_{1}+\mathbf{k}_{2}}^{f}\right\rangle \\
& \times\left(\left\langle n_{\mathbf{k}_{1}}^{c}\right\rangle-\left\langle n_{\mathbf{k}_{2}}^{c}\right\rangle\right) \\
- & \frac{1}{N^{2}} \sum_{\mathbf{k}_{1} \mathbf{k}_{2}} U_{\mathbf{k}_{1} \mathbf{k}_{2} \mathbf{k}, \lambda} \tilde{\alpha}_{\mathbf{k}_{2} \mathbf{k}_{1}, \mathbf{k}+\mathbf{k}_{1}-\mathbf{k}_{2}, \lambda} \\
& \times\left(1-\left\langle n_{\mathbf{k}+\mathbf{k}_{1}-\mathbf{k}_{2}}^{f}\right\rangle\right)\left(\left\langle n_{\mathbf{k}_{1}}^{c}\right\rangle-\left\langle n_{\mathbf{k}_{2}}^{c}\right\rangle\right) \\
\frac{d \Delta_{\mathbf{k}, \lambda}}{d \lambda}=- & \frac{1}{N} \sum_{\mathbf{k}_{1}} \tilde{\alpha}_{\mathbf{k}_{1} \mathbf{k} \mathbf{k}, \lambda} \Delta_{\mathbf{k}_{1}, \lambda}\left(\left\langle n_{\mathbf{k}_{1}}^{f}\right\rangle-\left\langle n_{\mathbf{k}_{1}}^{c}\right\rangle\right) \\
& \frac{1}{N^{2}} \sum_{\mathbf{k}_{1} \mathbf{k}_{2}}\left\{U_{\mathbf{k}_{1} \mathbf{k}_{2} \mathbf{k}_{2}, \lambda} \tilde{\alpha}_{\mathbf{k k}_{1} \mathbf{k}_{1}, \lambda} d_{\mathbf{k}}\left(1-\left\langle n_{\mathbf{k}_{1}}^{c}\right\rangle\right)\right. \\
& +U_{\mathbf{k}_{1} \mathbf{k} \mathbf{k}, \lambda} \tilde{\alpha}_{\mathbf{k k}_{2} \mathbf{k}_{2}, \lambda} d_{\mathbf{k}_{1}}\left\langle n_{\mathbf{k}}^{c}\right\rangle \\
& -U_{\mathbf{k}_{1} \mathbf{k}_{2} \mathbf{k}, \lambda}\left[\tilde{\alpha}_{\mathbf{k}_{1}+\mathbf{k}-\mathbf{k}_{2}, \mathbf{k}_{1} \mathbf{k}_{2}, \lambda} d_{\mathbf{k}_{1}+\mathbf{k}-\mathbf{k}_{2}}\left\langle n_{\mathbf{k}}^{f}\right\rangle\right. \\
& \left.\left.+\tilde{\alpha}_{\mathbf{k}_{2} \mathbf{k}, \mathbf{k}_{1}+\mathbf{k}-\mathbf{k}_{2}, \lambda} d_{\mathbf{k}_{1}}\left(1-\left\langle n_{\mathbf{k}_{1}+\mathbf{k}-\mathbf{k}_{2}}^{f}\right\rangle\right)\right]\right\}
\end{aligned}
$$

Here, we have defined expectation values

$$
\left\langle n_{\mathbf{k}}^{c}\right\rangle=\left\langle c_{\mathbf{k}}^{\dagger} c_{\mathbf{k}}\right\rangle, \quad\left\langle n_{\mathbf{k}}^{f}\right\rangle=\left\langle f_{\mathbf{k}}^{\dagger} f_{\mathbf{k}}\right\rangle, \quad d_{\mathbf{k}}=\left\langle c_{\mathbf{k}}^{\dagger} f_{\mathbf{k}}\right\rangle,
$$

which are formed with the full Hamiltonian. There is also an additional renormalization equation for the $\lambda$ dependent coupling $U_{\mathbf{k}_{1} \mathbf{k}_{2} \mathbf{k}, \lambda}$. It reads

$$
\frac{d U_{\mathbf{k}_{1} \mathbf{k}_{2} \mathbf{k}, \lambda}}{d \lambda}=\tilde{\eta}_{\mathbf{k}_{1} \mathbf{k}_{2} \mathbf{k}, \lambda} \tilde{\alpha}_{\mathbf{k}_{1} \mathbf{k}_{2} \mathbf{k}, \lambda} .
$$

Integrating the whole set of differential equations with the initial values given by Eq. (25), the completely renormalized Hamiltonian $\tilde{\mathcal{H}}:=\mathcal{H}_{\lambda \rightarrow 0}=\mathcal{H}_{0, \lambda \rightarrow 0}$ is obtained

$$
\begin{aligned}
\tilde{\mathcal{H}}= & \sum_{\mathbf{k}} \tilde{\varepsilon}_{\mathbf{k}}^{c}: c_{\mathbf{k}}^{\dagger} c_{\mathbf{k}}:+\sum_{\mathbf{k}} \tilde{\varepsilon}_{\mathbf{k}}^{f}: f_{\mathbf{k}}^{\dagger} f_{\mathbf{k}}: \\
& +\sum_{\mathbf{k}}\left(\tilde{\Delta}_{\mathbf{k}}: f_{\mathbf{k}}^{\dagger} c_{\mathbf{k}}:+ \text { H.c. }\right)
\end{aligned}
$$

where the quantities with tilde sign denote the parameter values at $\lambda \rightarrow 0$. The final Hamiltonian (35) can be diagonalized by use of a Bogoliubov transformation ${ }^{31}$ :

$$
\tilde{\mathcal{H}}=\sum_{\mathbf{k}} E_{\mathbf{k}}^{c}: \bar{c}_{\mathbf{k}}^{\dagger} \bar{c}_{\mathbf{k}}:+\sum_{\mathbf{k}} E_{\mathbf{k}}^{f}: \bar{f}_{\mathbf{k}}^{\dagger} \bar{f}_{\mathbf{k}}:+\tilde{E}
$$


Here, $\bar{c}_{\mathbf{k}}^{\dagger}$ and $\bar{f}_{\mathbf{k}}^{\dagger}$ are the new quasiparticle operators

$$
\begin{aligned}
& \bar{c}_{\mathbf{k}}^{\dagger}=u_{\mathbf{k}} c_{\mathbf{k}}^{\dagger}+v_{\mathbf{k}} f_{\mathbf{k}}^{\dagger}, \\
& \bar{f}_{\mathbf{k}}^{\dagger}=-v_{\mathbf{k}} c_{\mathbf{k}}^{\dagger}+u_{\mathbf{k}} f_{\mathbf{k}}^{\dagger},
\end{aligned}
$$

with

$$
\begin{aligned}
& u_{\mathbf{k}}^{2}=\frac{1}{2}\left(1+\operatorname{sgn}\left(\tilde{\varepsilon}_{\mathbf{k}}^{f}-\tilde{\varepsilon}_{\mathbf{k}}^{c}\right) \frac{\tilde{\varepsilon}_{\mathbf{k}}^{f}-\tilde{\varepsilon}_{\mathbf{k}}^{c}}{W_{\mathbf{k}}}\right), \\
& v_{\mathbf{k}}^{2}=\frac{1}{2}\left(1-\operatorname{sgn}\left(\tilde{\varepsilon}_{\mathbf{k}}^{f}-\tilde{\varepsilon}_{\mathbf{k}}^{c}\right) \frac{\tilde{\varepsilon}_{\mathbf{k}}^{f}-\tilde{\varepsilon}_{\mathbf{k}}^{c}}{W_{\mathbf{k}}}\right) .
\end{aligned}
$$

The quasiparticle energies are given by

$$
\begin{aligned}
& E_{\mathbf{k}}^{c}=\frac{\tilde{\varepsilon}_{\mathbf{k}}^{c}+\tilde{\varepsilon}_{\mathbf{k}}^{f}}{2}-\frac{\operatorname{sgn}\left(\tilde{\varepsilon}_{\mathbf{k}}^{f}-\tilde{\varepsilon}_{\mathbf{k}}^{c}\right)}{2} W_{\mathbf{k}}, \\
& E_{\mathbf{k}}^{f}=\frac{\tilde{\varepsilon}_{\mathbf{k}}^{c}+\tilde{\varepsilon}_{\mathbf{k}}^{f}}{2}+\frac{\operatorname{sgn}\left(\tilde{\varepsilon}_{\mathbf{k}}^{f}-\tilde{\varepsilon}_{\mathbf{k}}^{c}\right)}{2} W_{\mathbf{k}},
\end{aligned}
$$

where

$$
W_{\mathbf{k}}=\sqrt{\left(\tilde{\varepsilon}_{\mathbf{k}}^{c}-\tilde{\varepsilon}_{\mathbf{k}}^{f}\right)^{2}+4\left|\tilde{\Delta}_{\mathbf{k}}\right|^{2}} .
$$

\section{Expectation values}

The expectation values (33) in the set of renormalization equations have to be evaluated self-consistently. According to relation (14), thereby the same unitary transformation as for the Hamiltonian has to be used. For instance, following Eq. (14), the expectation value $\left\langle n_{\mathbf{k}}^{c}\right\rangle$ can be expressed by

$$
\left\langle n_{\mathbf{k}}^{c}\right\rangle=\left\langle c_{\mathbf{k}}^{\dagger} c_{\mathbf{k}}\right\rangle=\left\langle c_{\mathbf{k}}^{\dagger}(\lambda \rightarrow 0) c_{\mathbf{k}}(\lambda \rightarrow 0)\right\rangle_{\tilde{\mathcal{H}}},
$$

where the average on the r.h.s. is formed with the fully renormalized Hamiltonian $\tilde{\mathcal{H}}$, and $c_{\mathbf{k}}^{\dagger}(\lambda)$ is given by $c_{\mathbf{k}}^{\dagger}(\lambda)=e^{\mathcal{X}} c_{\mathbf{k}}^{\dagger} e^{-\mathcal{X}_{\lambda}}$. For the transformed operator we use as ansatz

$$
\begin{aligned}
: c_{\mathbf{k}}^{\dagger}(\lambda):= & x_{\mathbf{k}, \lambda}: c_{\mathbf{k}}^{\dagger}: \\
& +\frac{1}{N} \sum_{\mathbf{k}_{1} \mathbf{k}_{2}} y_{\mathbf{k}_{1} \mathbf{k k}_{2}, \lambda}: c_{\mathbf{k}_{1}}^{\dagger} f_{\mathbf{k}_{2}}^{\dagger} f_{\mathbf{k}_{1}+\mathbf{k}_{2}-\mathbf{k}}: .
\end{aligned}
$$

with a coherent part $\propto x_{\mathbf{k}, \lambda}$ and an incoherent part $\propto y_{\mathbf{k}_{1} \mathbf{k}_{2}, \lambda}$. The operator structure in (45) is again taken over from the lowest order expansion of the unitary transformation. For the $\lambda$-dependent coefficients $x_{\mathbf{k}, \lambda}$ and $y_{\mathbf{k}_{1} \mathbf{k k}_{2}, \lambda}$ new renormalization equations can be derived. They read

$$
\begin{aligned}
\frac{d x_{\mathbf{k}, \lambda}}{d \lambda} & =-\frac{1}{N^{2}} \sum_{\mathbf{k}_{1} \mathbf{k}_{2}} y_{\mathbf{k}_{1} \mathbf{k}_{2}, \lambda} \tilde{\alpha}_{\mathbf{k k}_{1}, \mathbf{k}_{1}+\mathbf{k}_{2}-\mathbf{k}, \lambda}\left[\left(1-\left\langle n_{\mathbf{k}_{1}}^{c}\right\rangle\right)\right. \\
& \left.\times\left(\left\langle n_{\mathbf{k}_{1}+\mathbf{k}_{2}-\mathbf{k}}^{f}\right\rangle-\left\langle n_{\mathbf{k}_{2}}^{f}\right\rangle\right)+\left\langle n_{\mathbf{k}_{2}}^{f}\right\rangle\left(1-\left\langle n_{\mathbf{k}_{1}+\mathbf{k}_{2}-\mathbf{k}}^{f}\right\rangle\right)\right],
\end{aligned}
$$

$\frac{d y_{\mathbf{k}_{1} \mathbf{k k}_{2}, \lambda}}{d \lambda}=-x_{\mathbf{k}, \lambda} \tilde{\alpha}_{\mathbf{k}_{1} \mathbf{k k}_{2}, \lambda}$.
Integration between $\Lambda$ (where $x_{\mathbf{k}, \Lambda}=1$ and $y_{\mathbf{k}_{1} \mathbf{k k}_{2}, \Lambda}=0$ ) and $\lambda=0$ leads to

$$
\begin{aligned}
: c_{\mathbf{k}}^{\dagger}(\lambda \rightarrow 0):=\tilde{x}_{\mathbf{k}}: c_{\mathbf{k}}^{\dagger}: \\
+\frac{1}{N} \sum_{\mathbf{k}_{1} \mathbf{k}_{2}} \tilde{y}_{\mathbf{k}_{1} \mathbf{k k}_{2}}: c_{\mathbf{k}_{1}}^{\dagger} f_{\mathbf{k}_{2}}^{\dagger} f_{\mathbf{k}_{1}+\mathbf{k}_{2}-\mathbf{k}}:
\end{aligned}
$$

from which $\left\langle n_{\mathbf{k}}^{c}\right\rangle$ is found

$$
\begin{aligned}
\left\langle n_{\mathbf{k}}^{c}\right\rangle= & \left|\tilde{x}_{\mathbf{k}}\right|^{2}\left\langle c_{\mathbf{k}}^{\dagger} c_{\mathbf{k}}\right\rangle_{\tilde{\mathcal{H}}} \\
& +\frac{1}{N^{2}} \sum_{\mathbf{k}_{1} \mathbf{k}_{2}}\left|\tilde{y}_{\mathbf{k}_{1} \mathbf{k}_{2}}\right|^{2}\left\langle c_{\mathbf{k}_{1}}^{\dagger} c_{\mathbf{k}_{1}}\right\rangle_{\tilde{\mathcal{H}}}\left\langle f_{\mathbf{k}_{2}}^{\dagger} f_{\mathbf{k}_{2}}\right\rangle_{\tilde{\mathcal{H}}} \\
& \times\left(1-\left\langle f_{\mathbf{k}_{1}+\mathbf{k}_{2}-\mathbf{k}}^{\dagger} f_{\mathbf{k}_{1}+\mathbf{k}_{2}-\mathbf{k}}\right\rangle_{\tilde{\mathcal{H}}}\right) .
\end{aligned}
$$

The remaining expectation values $\left\langle n_{\mathbf{k}}^{f}\right\rangle$ and $d_{\mathbf{k}}$ can be evaluated by using an equivalent ansatz for : $f_{\mathbf{k}}^{\dagger}(\lambda)$ :,

$$
\begin{aligned}
& : f_{\mathbf{k}}^{\dagger}(\lambda):=x_{\mathbf{k}, \lambda}^{\prime}: f_{\mathbf{k}}^{\dagger}: \\
& \quad+\frac{1}{N} \sum_{\mathbf{k}_{1} \mathbf{k}_{2}} y_{\mathbf{k}_{1} \mathbf{k}_{2}, \mathbf{k}-\mathbf{k}_{1}+\mathbf{k}_{2}, \lambda}^{\prime}: c_{\mathbf{k}_{1}}^{\dagger} c_{\mathbf{k}_{2}} f_{\mathbf{k}-\mathbf{k}_{1}+\mathbf{k}_{2}}^{\dagger}:,
\end{aligned}
$$

consisting again of a coherent and an incoherent part with $\lambda$-dependent coefficients $x_{\mathbf{k}, \lambda}^{\prime}$ and $y_{\mathbf{k}_{1} \mathbf{k}_{2}, \mathbf{k}-\mathbf{k}_{1}+\mathbf{k}_{2}, \lambda}^{\prime}$, respectively. Their renormalization equations read

$$
\begin{aligned}
\frac{d x_{\mathbf{k}, \lambda}^{\prime}}{d \lambda}= & -\frac{1}{N^{2}} \sum_{\mathbf{k}_{1} \mathbf{k}_{2}} y_{\mathbf{k}_{1} \mathbf{k}_{2}, \mathbf{k}-\mathbf{k}_{1}+\mathbf{k}_{2}, \lambda}^{\prime} \tilde{\alpha}_{\mathbf{k}_{2} \mathbf{k}_{1} \mathbf{k}, \lambda}\left[\left\langle n_{\mathbf{k}-\mathbf{k}_{1}+\mathbf{k}_{2}}^{f}\right\rangle\right. \\
& \left.\times\left(\left\langle n_{\mathbf{k}_{1}}^{c}\right\rangle-\left\langle n_{\mathbf{k}_{2}}^{c}\right\rangle\right)+\left\langle n_{\mathbf{k}_{2}}^{c}\right\rangle\left(1-\left\langle n_{\mathbf{k}_{1}}^{c}\right\rangle\right)\right], \quad(51)
\end{aligned}
$$

$\frac{d y_{\mathbf{k}_{1} \mathbf{k}_{2}, \mathbf{k}-\mathbf{k}_{1}+\mathbf{k}_{2}, \lambda}^{\prime}}{d \lambda}=-x_{\mathbf{k}, \lambda}^{\prime} \tilde{\alpha}_{\mathbf{k}_{1} \mathbf{k}_{2}, \mathbf{k}-\mathbf{k}_{1}+\mathbf{k}_{2}, \lambda}$,

where the initial values are $x_{\mathbf{k}, \Lambda}^{\prime}=1$ and $y_{\mathbf{k}_{1} \mathbf{k}_{2}, \mathbf{k}-\mathbf{k}_{1}+\mathbf{k}_{2}, \Lambda}^{\prime}=0$. Similar to Eq. (49), we are led to

$$
\begin{aligned}
\left\langle n_{\mathbf{k}}^{f}\right\rangle= & \left|\tilde{x}_{\mathbf{k}}^{\prime}\right|^{2}\left\langle f_{\mathbf{k}}^{\dagger} f_{\mathbf{k}}\right\rangle_{\tilde{\mathcal{H}}} \\
+ & \frac{1}{N^{2}} \sum_{\mathbf{k}_{1} \mathbf{k}_{2}}\left|\tilde{y}_{\mathbf{k}_{1} \mathbf{k}_{2}, \mathbf{k}-\mathbf{k}_{1}+\mathbf{k}_{2}}^{\prime}\right|^{2}\left\langle f_{\mathbf{k}-\mathbf{k}_{1}+\mathbf{k}_{1}}^{\dagger} f_{\mathbf{k}-\mathbf{k}_{1}+\mathbf{k}_{2}}\right\rangle_{\tilde{\mathcal{H}}} \\
& \times\left\langle c_{\mathbf{k}_{1}}^{\dagger} c_{\mathbf{k}_{1}}\right\rangle_{\tilde{\mathcal{H}}}\left(1-\left\langle c_{\mathbf{k}_{2}}^{\dagger} c_{\mathbf{k}_{2}}\right\rangle_{\tilde{\mathcal{H}}}\right) \\
d_{\mathbf{k}}= & \tilde{x}_{\mathbf{k}} \tilde{x}_{\mathbf{k}}^{\prime}\left\langle f_{\mathbf{k}}^{\dagger} c_{\mathbf{k}}\right\rangle_{\tilde{\mathcal{H}}} \\
- & \frac{1}{N^{2}} \sum_{\mathbf{k}_{1} \mathbf{k}_{2}} \tilde{y}_{\mathbf{k}_{1} \mathbf{k}_{2}, \mathbf{k}-\mathbf{k}_{1}+\mathbf{k}_{2}}^{\prime} \tilde{y}_{\mathbf{k}_{1} \mathbf{k}, \mathbf{k}-\mathbf{k}_{1}+\mathbf{k}_{2}}\left\langle c_{\mathbf{k}_{1}}^{\dagger} c_{\mathbf{k}_{1}}\right\rangle_{\tilde{\mathcal{H}}} \\
& \times\left\langle f_{\mathbf{k}_{2}}^{\dagger} c_{\mathbf{k}_{2}}\right\rangle_{\tilde{\mathcal{H}}}\left\langle f_{\mathbf{k}-\mathbf{k}_{1}+\mathbf{k}_{2}}^{\dagger} f_{\mathbf{k}-\mathbf{k}_{1}+\mathbf{k}_{2}}\right\rangle_{\tilde{\mathcal{H}}}
\end{aligned}
$$

In the last step, one has to evaluate the expectation values on the r.h.sides of Eqs. (49), (53), and (54), which are formed with $\tilde{\mathcal{H}}$. Using the diagonal form of $\tilde{\mathcal{H}}$ in one easily finds 31

$$
\begin{aligned}
&\left\langle c_{\mathbf{k}}^{\dagger} c_{\mathbf{k}}\right\rangle_{\tilde{\mathcal{H}}}=u_{\mathbf{k}}^{2} f\left(E_{\mathbf{k}}^{c}\right)+v_{\mathbf{k}}^{2} f\left(E_{\mathbf{k}}^{f}\right), \\
&\left\langle f_{\mathbf{k}}^{\dagger} f_{\mathbf{k}}\right\rangle_{\tilde{\mathcal{H}}}=v_{\mathbf{k}}^{2} f\left(E_{\mathbf{k}}^{c}\right)+u_{\mathbf{k}}^{2} f\left(E_{\mathbf{k}}^{f}\right), \\
&\left\langle f_{\mathbf{k}}^{\dagger} c_{\mathbf{k}}\right\rangle_{\tilde{\mathcal{H}}}=-\left[f\left(E_{\mathbf{k}}^{c}\right)-f\left(E_{\mathbf{k}}^{f}\right)\right] \operatorname{sgn}\left(\tilde{\varepsilon}_{\mathbf{k}}^{f}-\tilde{\varepsilon}_{\mathbf{k}}^{c}\right) \frac{\tilde{\Delta}_{\mathbf{k}}}{W_{\mathbf{k}}},
\end{aligned}
$$


where $f\left(E_{\mathbf{k}}^{\eta}\right)$ is the Fermi function.

\section{Spectral functions}

Let us consider the one-particle spectral function for $c$ electrons

$$
A^{c}(\mathbf{k}, \omega)=-\frac{1}{\pi} \operatorname{Im} G^{c}(\mathbf{k}, \omega),
$$

where $G^{c}(\mathbf{k}, \omega)$ is the Fourier transform of the retarded Green function

$$
G^{c}(\mathbf{k}, \omega)=\left\langle\left\langle c_{\mathbf{k}} ; c_{\mathbf{k}}^{\dagger}\right\rangle\right\rangle\left(\omega+i 0^{+}\right) .
$$

Using again relation (14), the Green function can be rewritten

$$
\left\langle\left\langle c_{\mathbf{k}} ; c_{\mathbf{k}}^{\dagger}\right\rangle\right\rangle(\omega)=\left\langle\left\langle c_{\mathbf{k}}(\lambda \rightarrow 0) ; c_{\mathbf{k}}^{\dagger}(\lambda \rightarrow 0)\right\rangle\right\rangle_{\tilde{\mathcal{H}}}(\omega),
$$

where the expectation value on the r.h.side is again formed with $\tilde{\mathcal{H}}$. Using expression (48) for $c_{\mathbf{k}}^{\dagger}(\lambda \rightarrow 0)$, we are immediately led the following result for the $c$-electron spectral function

$$
\begin{aligned}
& A^{c}(\mathbf{k}, \omega)=\left|\tilde{x}_{\mathbf{k}}\right|^{2}\left[u_{\mathbf{k}}^{2} \delta\left(\omega-E_{\mathbf{k}}^{c}\right)+v_{\mathbf{k}}^{2} \delta\left(\omega-E_{\mathbf{k}}^{f}\right)\right] \\
& +\frac{1}{N^{2}} \sum_{\mathbf{k}_{1} \mathbf{k}_{2}}\left|\tilde{y}_{\mathbf{k}_{1} \mathbf{k k}_{2}}\right|^{2} \delta\left(\omega-\left(E_{\mathbf{k}_{1}}^{c}-E_{\mathbf{k}_{1}+\mathbf{k}_{2}-\mathbf{k}}^{f}+E_{\mathbf{k}_{2}}^{f}\right)\right) \\
& \quad \times\left[\left\langle c_{\mathbf{k}_{1}}^{\dagger} c_{\mathbf{k}_{1}}\right\rangle_{\tilde{\mathcal{H}}}\left(\left\langle f_{\mathbf{k}_{2}}^{\dagger} f_{\mathbf{k}_{2}}\right\rangle_{\tilde{\mathcal{H}}}-\left\langle f_{\mathbf{k}_{1}+\mathbf{k}_{2}-\mathbf{k}}^{\dagger} f_{\mathbf{k}_{1}+\mathbf{k}_{2}-\mathbf{k}}\right\rangle_{\tilde{\mathcal{H}}}\right)\right. \\
& \left.\quad+\left\langle f_{\mathbf{k}_{1}+\mathbf{k}_{2}-\mathbf{k}}^{\dagger} f_{\mathbf{k}_{1}+\mathbf{k}_{2}-\mathbf{k}}\right\rangle_{\tilde{\mathcal{H}}}\left(1-\left\langle f_{\mathbf{k}_{2}}^{\dagger} f_{\mathbf{k}_{2}}\right\rangle_{\tilde{\mathcal{H}}}\right)\right] .
\end{aligned}
$$

Note that we have restricted ourselves to the leading order in the EI order parameter. In the same way, we can also calculate the spectral function $A^{f}(\mathbf{k}, \omega)$ for the $f$ electrons. The final result reads

$$
\begin{aligned}
& A^{f}(\mathbf{k}, \omega)=\left|\tilde{x}_{\mathbf{k}}^{\prime}\right|^{2}\left[v_{\mathbf{k}}^{2} \delta\left(\omega-E_{\mathbf{k}}^{c}\right)+u_{\mathbf{k}}^{2} \delta\left(\omega-E_{\mathbf{k}}^{f}\right)\right] \\
& +\frac{1}{N^{2}} \sum_{\mathbf{k}_{1} \mathbf{k}_{2}}\left|\tilde{y}_{\mathbf{k}_{1} \mathbf{k}_{2}, \mathbf{k}-\mathbf{k}_{1}+\mathbf{k}_{2}}^{\prime}\right|^{2} \\
& \times \delta\left[\omega-\left(E_{\mathbf{k}-\mathbf{k}_{1}+\mathbf{k}_{2}}^{f}-E_{\mathbf{k}_{2}}^{c}+E_{\mathbf{k}_{1}}^{c}\right)\right] \\
& \times\left[\left\langle c_{\mathbf{k}_{1}}^{\dagger} c_{\mathbf{k}_{1}}\right\rangle_{\tilde{\mathcal{H}}}\left(1-\left\langle c_{\mathbf{k}_{2}}^{\dagger} c_{\mathbf{k}_{2}}\right\rangle_{\tilde{\mathcal{H}}}\right)\right. \\
& \quad+\left(\left\langle c_{\mathbf{k}_{2}}^{\dagger} c_{\mathbf{k}_{2}}\right\rangle_{\tilde{\mathcal{H}}}-\left\langle c_{\mathbf{k}_{1}}^{\dagger} c_{\mathbf{k}_{1}}\right\rangle_{\tilde{\mathcal{H}}}\right) \\
& \left.\quad \times\left(1-\left\langle f_{\mathbf{k}-\mathbf{k}_{1}+\mathbf{k}_{2}}^{\dagger} f_{\mathbf{k}-\mathbf{k}_{1}+\mathbf{k}_{2}}\right\rangle_{\tilde{\mathcal{H}}}\right)\right]
\end{aligned}
$$

\section{NUMERICAL RESULTS AND DISCUSSION}

We now will evaluate the analytical expressions of the PRM approach outlined so far. Of course, the set of equations (49), (53), and (54) has to be solved numerically. To this end, we choose some initial values for $\left\langle n_{\mathbf{k}}^{c}\right\rangle$, $\left\langle n_{\mathbf{k}}^{f}\right\rangle$, and $d_{\mathbf{k}}$ (assuming $\left.\left\langle c_{\mathbf{k}}^{\dagger} f_{\mathbf{k}}\right\rangle=\left\langle f_{\mathbf{k}}^{\dagger} c_{\mathbf{k}}\right\rangle\right)$, and determine the renormalization of the Hamiltonian and all operators, by solving the differential equations (30)-(32), (47), and (52). Performing the limit $\lambda \rightarrow 0$, all model parameters will be renormalized. Then, using $\tilde{\mathcal{H}}$, the new expectation values [Eqs. (55)-(57)] are calculated, and the renormalization process of the Hamiltonian is restarted. Convergence is assumed to be achieved if all quantities are determined with a relative error less than $10^{-5}$. The dynamical correlation functions (61)-62) are evaluated using a Gaussian broadening in energy space of width 0.06. Because of the large number of differential equations that have to be solved, we confine ourselves, in what follows, to the investigation of the 1D case and limit the number of lattice sites ( $k$-points) to $N=60$.

\section{A. Ground-state properties}

\section{Order parameter}

We begin by scanning the parameter space of the $1 \mathrm{D}$ EFKM, in order to detect an EI ground state. The $T=0$ quantum phase diagram of the 1D EFKM has been previously explored by the CPMC technique ${ }^{32}$, after mapping the EFKM - rewritten in pseudospin variables - into a negative $U$ asymmetric Hubbard model with the Zeeman term replaced by a chemical potential ${ }^{14}$. Thereby, in terms of our original language, a transition from a mixed-valence regime to a nonmixed valence regime was observed, which corresponds to the transition from the EI to a band insulator. In $1 \mathrm{D}$, the EI phase is characterized by critical excitonic correlations. Surprisingly the topology of the 1D phase diagram is the same as for the $2 \mathrm{D}$ and $3 \mathrm{D}$ cases, which were studied by CPMC 14 (2D) and Hartree-Fock ${ }^{16.17}$ (2D, 3D) approaches. To benchmark the reliability of the PRM, we have compared the 1D PRM EI band-insulator transition points with those obtained by the rather unbiased CPMC method, and found excellent agreement. For example, we obtain $\varepsilon_{c, \mathrm{PRM}}^{f}=-1.81 \simeq \varepsilon_{c, \mathrm{CPMC}}^{f}=-1.80$ for $t^{f}=-0.3$, $U=1$ (cf. Fig. 1 from Ref. 14).

Figures 2 and 3 show the onset of the EI phase for different values of $U\left(t^{f}=-0.3\right.$ fixed) and $t^{f}(U=0.8$ fixed), respectively, as the $f$-electron level is varied (see main panels). Obviously, the EI phase emerges above a critical Coulomb attraction strength $U_{c 1}$ (cf. inset Fig. (2), provided that $c$ and $f$ bands overlap. Moving up the $\varepsilon^{f}$ level, the top of the $f$ band reaches the bottom of the $c$ band at a critical value $\varepsilon_{c}^{f}$. Then some $f$ electrons can be transferred into $c$-band electrons and exciton bound-states of $f$-band holes and $c$-band electrons may form if the Coulomb attraction is sufficiently strong. Recent Hartree-Fock- and slave-boson-theory-based studies $17,18,21$ yield a second, upper critical value of the Coulomb attraction $U_{c 2}$, such that the EI phase is confined in between $U_{c 1}$ and $U_{c 2}$. The PRM data, produced up to now, will rather not confirm this controversial find- 


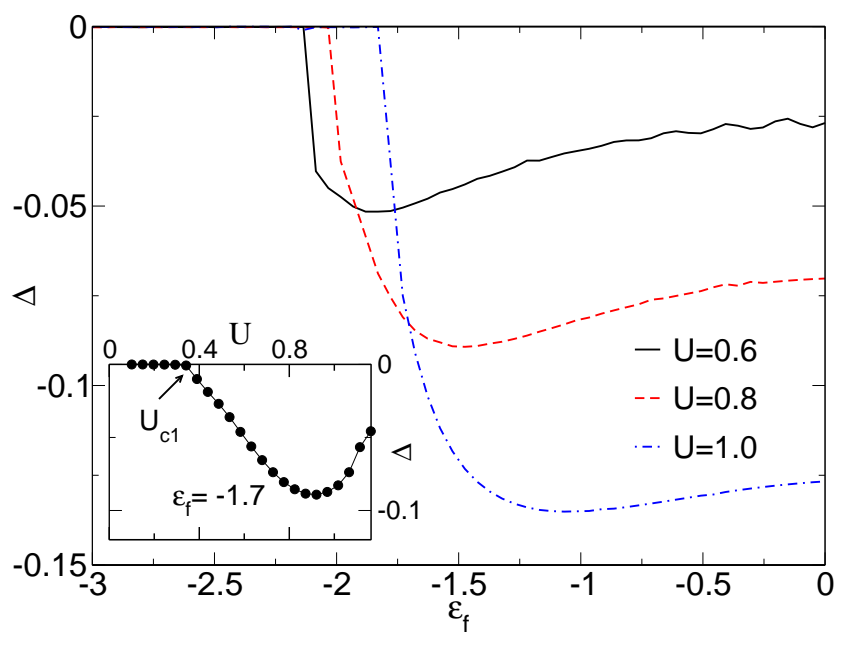

FIG. 2: (Color online) EI order parameter, $\Delta$, in the 1D EFKM. The $f$-electron transfer-integral is fixed to be $t^{f}=$ -0.3 (all energies are given in units of $t^{c}$ ). In what follows, we assume $\varepsilon^{c}=0$ without loss of generality. The main panel gives $\Delta$ as a function of the position of the $f$-electron level, $\varepsilon^{f}$, for different values of $U$, while the inset shows the variation of $\Delta$ with $U$ at $\varepsilon^{f}=-1.7$. The calculations were performed at basically zero temperature, $T=10^{-3}$.

ing (see inset Fig. 2). However in the large- $U$ limit, the numerical calculations are ill-conditioned and tedious, especially in $1 \mathrm{D}$. So the question whether $U_{c 2}=\infty$ remains open. That the appearance of the EI phase is intimately connected with the build-up of $f$-c electron coherence and a non-integral $f$-electron valence is demonstrated by the inset of Fig. [3] depicting $\left\langle n^{f}\right\rangle$ (cf. also the discussion of Fig. [5 below).

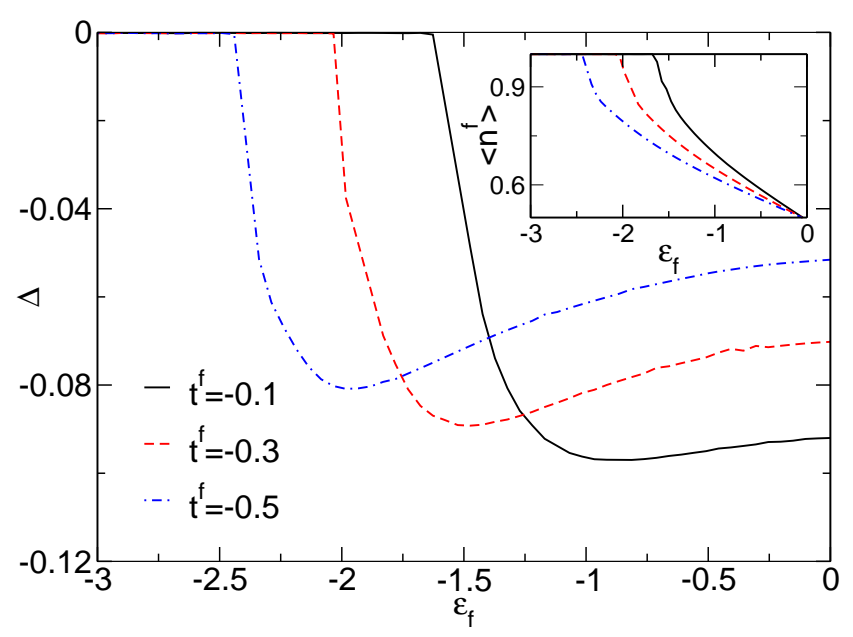

FIG. 3: (Color online) EI order parameter in the 1D EFKM. The main panel gives $\Delta$ as a function of the position of the $f$-electron level for different $t^{f}$ at $U=0.8$. The inset shows the corresponding variation of the mean $f$-electron number.
The physical picture developed so far does not change if the $f$-electron band approaches the $c$-electron band from above. That is our results are one and the same changing the sign of $\varepsilon^{f}$. We note that in a rather small region around $\varepsilon^{f}=0$ (symmetric band case), a chargedensity-wave state is assumed to be the true ground state, i.e., the EI phase becomes metastable. Focussing on the characterization of the EI phase we will not address this issue here.

\section{Band renormalization}

Next we investigate the renormalization of the 1D band structure. Figure 4 displays the $k$-dependence of the quasiparticle energies $E_{k}^{\eta}$ in the EI phase, where open and filled symbols correspond to bands having predominantly $c$ - and $f$-electron character, respectively. There are two features of importance. First, the band structure is clearly gapful in the EI phase. Hence, for the half-filled band case, the system is insulating. The gap originates from $c-f$ electron hybridization, induced by the attractive Coulomb interaction. Second, the lower and upper quasiparticle bands are narrowed as a result of the electronic correlations. While the maximum of the lower (at $T=0$ completely occupied) band is displaced to larger $k$-values as $U$ increases, the minimum of the upper (at $T=0$ empty) band moves to smaller $k$, accompanied by a flattening of the ' $f$-band' near $k=0$. At very large $U$ (not depicted), the gap is kept (but different in nature), because of the extreme Hartree shift, leading to a $c-f$ band-splitting. Except of contributions from the band narrowing, the Hartree shift to the quasiparticle energies is roughly given by the sum of $\varepsilon^{c}-\varepsilon^{f}$ and $U\left(\left\langle n^{f}\right\rangle-\left\langle n^{c}\right\rangle\right)$ (compare Eqs. (6), (41), (42)).

\section{Momentum distribution function}

Another quantity of interest is the occupation number of fermionic states carrying momentum $k$. For free fermions, at $T=0$, all states up to the Fermi energy, $E_{F}$, are occupied, so that the momentum distribution function, $n(k)=\left\langle n_{k}\right\rangle$, has a discontinuity at the corresponding Fermi momentum, $k_{F}$, where $n(k)$ jumps from one to zero. In an interacting Fermi liquid there is still a discontinuity, but the jump is less than one. In 1D, normally Luttinger-liquid behavior emerges, with an essential power-law singularity at $k_{F}$. For the insulating state, however, $n(k)$ is given by a smooth curve. This holds, e.g., for the charge-density-wave ground states of 1D $t-V$ and Holstein-type models ${ }^{33}$, and should also be valid for the EI phase in the 1D EFKM. Indeed, the momentum distribution functions of $c$ and $f$ electrons, $n^{\eta}(k)$, depicted in Fig. 5 confirm this picture. We see that $n^{f}(k)\left(n^{c}(k)\right)$ monotonously increases (decreases) as $k$ varies from $k=0$ to $k=\pi$. As expected, the drop (upturn) near ' $k_{F}$ ' softens at larger interactions strengths. 


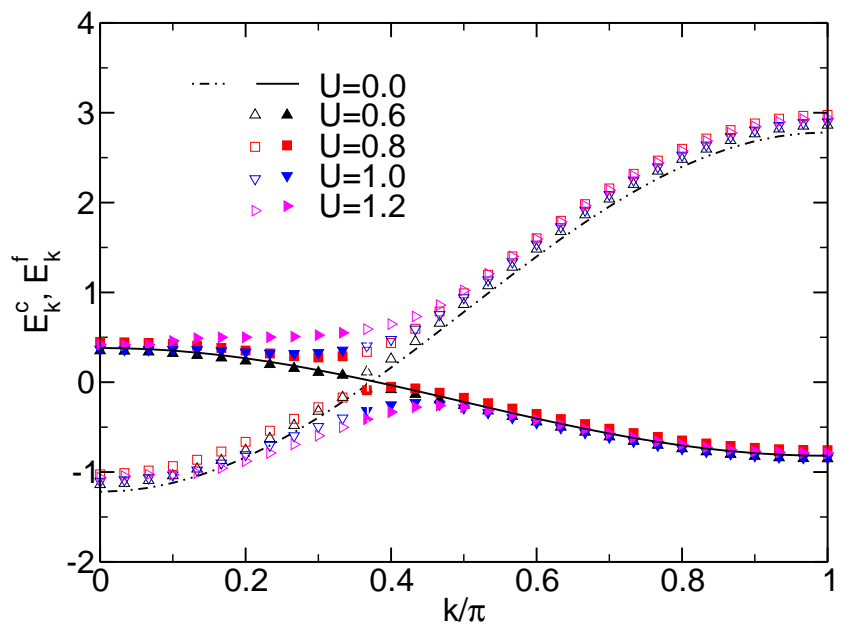

FIG. 4: (Color online) Renormalized quasiparticle band dispersion of $c$ electrons $\left(E_{k}^{c}\right.$, open symbols) and $f$ electrons $\left(E_{k}^{f}\right.$, filled symbols) in the EI phase of the 1D EFKM for different values of $U$. Again the 'bare' band structure is parameterized by $\varepsilon^{c}=0, \varepsilon^{f}=-1.0, t^{f}=-0.3$ (dot-dashed and solid lines). Note that the scale of the ordinate is shifted in order to fix the Fermi energy at zero energy.

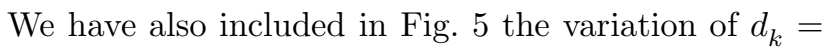
$\left\langle f_{k}^{\dagger} c_{k}\right\rangle$. This expectation value enters into the equation for the order parameter (5). In some sense, it can be taken as a measure of the range in $k$-space, where $c$ electrons and $f$-holes are involved in the exciton formation and condensation process. Having a $U$-driven BCS$\mathrm{BEC}$ crossover scenario in the EI phase of the EFKM in mind $^{9,21}$, the broadening of the distribution of $d_{k}$ with increasing $U$ might indicate the condensation of a more local two-body BEC-like bound-state out of a BCS-like Cooper-pair state. Note that in the BEC-like state the Fermi surface plays no role.

\section{B. Spectral properties}

In this section, we present first results for the photoemission (PE) spectra in the EI phase of the EFKM. The calculated single-particle spectral functions, associated with the emission (PE) or injection (inverse $\mathrm{PE}$ ) of an electron with wave vector $k$, directly measure the occupied and unoccupied densities of single-particle states, and therefore are well suited to investigate pairing gaps as well. Note that the ARPES spectral functions

$$
\begin{aligned}
A_{\mathrm{ARPES}}^{\eta}(\mathbf{k}, \omega) & =\frac{1}{2 \pi} \int_{-\infty}^{+\infty}\left\langle\eta_{\mathbf{k}}^{\dagger}(t) \eta_{\mathbf{k}}\right\rangle e^{-i \omega t} d t \\
& =\frac{1}{1+e^{\beta \omega}} A^{\eta}(\mathbf{k}, \omega)
\end{aligned}
$$

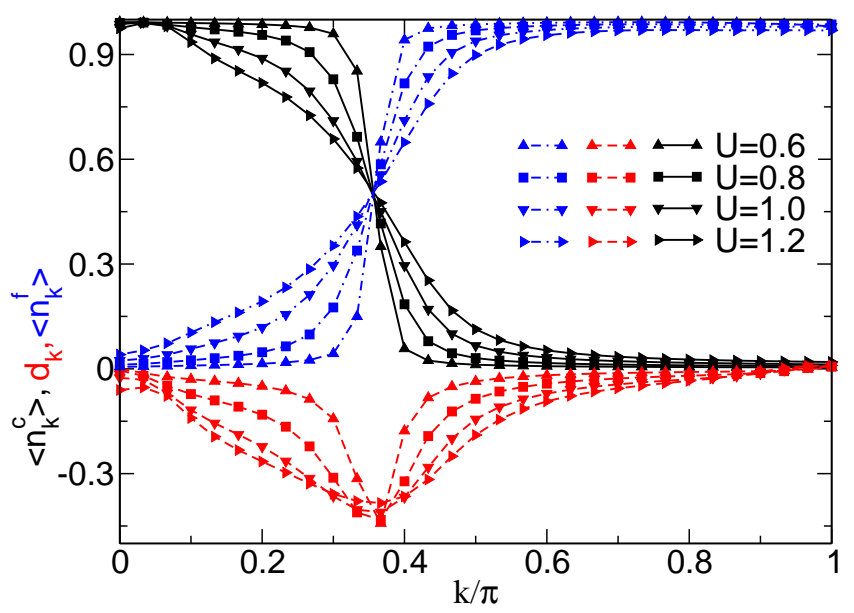

FIG. 5: (Color online) Momentum distribution functions $\left\langle n_{k}^{c}\right\rangle=\left\langle c_{k}^{\dagger} c_{k}\right\rangle$ (black solid lines) and $\left\langle n_{k}^{f}\right\rangle=\left\langle f_{k}^{\dagger} f_{k}\right\rangle$ (blue dash-dotted lines) for the same model parameters as used in Fig. 4. The red dashed lines show the corresponding 'order parameter' functions $d_{k}$, see Eq. (5).

fulfill at $T=0$ the frequency sum rule

$$
\int_{-\infty}^{0} A^{\eta}(\mathbf{k}, \omega) d \omega=\left\langle n_{\mathbf{k}}^{\eta}\right\rangle
$$

where $\left\langle n_{\mathbf{k}}^{\eta}\right\rangle$ is given by Eqs. (49) and (53) (cf. Fig. (5).

Figure [6] displays the zero-temperature, wave-vector and energy resolved single-particle spectral functions, $A^{\eta}(k, \omega)$ [see Eqs. (61), (62)], for a bare band structure parameterized by $\varepsilon^{f}=-1\left(\varepsilon^{c}=0\right), t^{f}=-0.3\left(t^{c}=1\right)$. For weak Coulomb attraction, $U=0.2$ (upper panels) we are still in the semi-metallic phase, and consistently $A^{c}(k, \omega)$ and $A^{f}(k, \omega)$ follow the nearly unrenormalized $c$ - and $f$-band dispersions, respectively. Concomitantly, we find a more or less uniform distribution of the spectral weight and negligible incoherent contributions (see righthand panels for $A^{c}(k, \omega)$ and $\left.A^{f}(k, \omega)\right)$. When entering the EI phase by increasing $U$ to $U=0.6$, a gap feature develops at the Fermi energy (Fermi momentum), but away from that the spectra still show the main characteristics of the semi-metallic state (cf. both middle panels). At a still larger value $U=1.2$, the gap broadens. Most notably, however, is a significant redistribution of the spectral weight from the coherent to the incoherent part of $A^{\eta}(k, \omega)$, with pronounced absorption maxima at $k=0, \pi$. Of particular importance is a considerable admixture of $c$-electron contributions to the $f$-electron spectrum in an interval between $k=0$ and $k \simeq k_{F}$. The resulting double peak structure around the Fermi level can be considered as an almost $k$-independent bound object of $c$ electrons and $f$ holes.

If we change the location of the $f$-band by lowering the position of the $f$-electron level, we can achieve that the band structure becomes gapful due to the Hartree shift (6), even for moderate values of $U$ (assuming $\Delta \equiv$ 

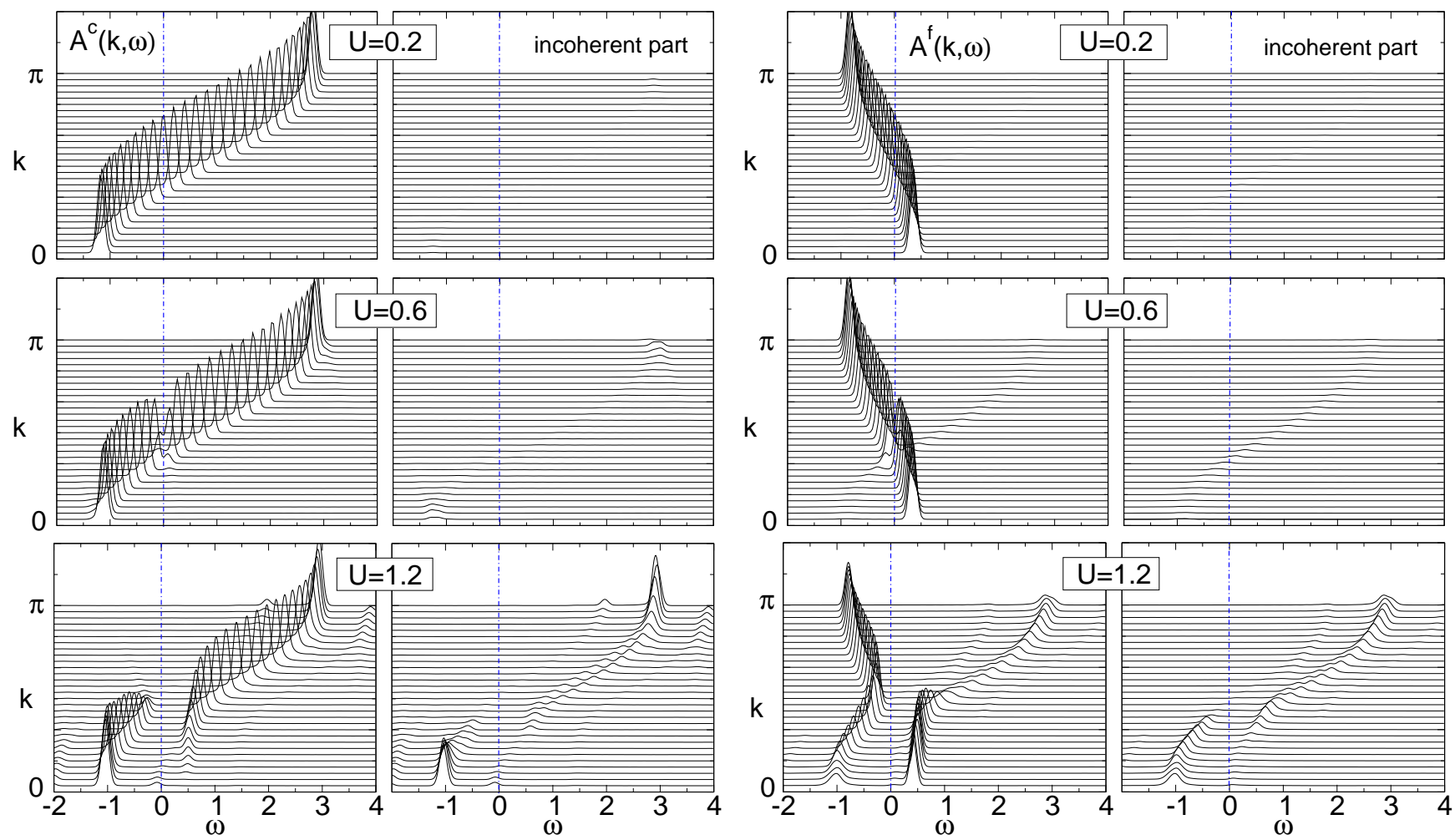

FIG. 6: (Color online) Wave-number resolved photoemission spectra of the 1D half-filled EFKM. The $c$ - (left-hand panels) and $f$-electron (right-hand panels) single-particle spectral functions were calculated for several characteristic values of $U$ at $\varepsilon^{f}=-1.0, t^{f}=-0.3, T=10^{-3}$. The left panels show in each case the total spectra, $A^{\eta}(k, \omega)$, whereas the right panels give the 'incoherent' contributions only (second term in Eqs. (61), (62)). The vertical dot-dashed lines mark the chemical potential.

$0)$. For example, at $\varepsilon^{f}=-1.7$ and $t^{f}=-0.3$, a $c$ $f$ band-splitting (positive Hartree gap $\Delta_{H}$, cf. Fig. 1) occurs already at $U_{H} \simeq 0.94$. Such a situation comes closer to the $\mathrm{Tm}[\mathrm{Se}, \mathrm{Te}]$ system $^{8}$. Figure 7 shows the PE spectra calculated for these parameter values. Compared to the case $\varepsilon^{f}=-1$, for $U=0.6$ the gap is clearly a bit more shaped, but the main features of the spectrum stay the same. Here we are in the BCS-regime, where pairing fluctuations are expected to be small. For $U=$ $1.2>U_{H}$, we enter the BEC-regime, where preformed pairs acquire quantum coherence (many-body character) during the condensation process. Since $\Delta \neq 0$ the gap persists. The distinct incoherent contributions, showing up in the $A^{\eta}(k, \omega)$ spectra at high energies, are related to the dissociation of two-particle bound-states (excitons).

To visualize more clearly the spectral weight and line-shape of the various absorption signals, we provide in Fig. 8 a color-map intensity-plot of the singleparticle spectral functions depicted in Fig. 7 Particularly from the $A^{f}(k, \omega)$ data, it appears that the BCS-BEC crossover is again marked by a notable admixture of $c$ electron-like contributions leading to new peak-line structures in the spectrum (cf. right-hand panels of Fig. 8).

\section{CONCLUSIONS}

To summarize, we adapted a continuous version of the projective renormalization method to the extended Falicov-Kimball model to examine the possible existence of a collective excitonic insulator phase at the semimetalsemiconductor transition. Thereby the PRM approach allows us to derive analytical expressions for the EI order parameter and various other physical quantities characterizing the ground-state and spectral properties of the model. The self-consistent evaluation of the renormalization equations yields a stable EI solution for the onedimensional EFKM, at half-filling and zero temperature. It therefore confirms previous, straight numerical data by constrained path integral Monte Carlo. In particular, the phase boundary between the excitonic and band insulator agrees even quantitatively with the CPMC results. Thus, increasing the Coulomb attraction between $c$-band electrons and $f$-band holes, the appearance of a semimetal-EI transition seems to be settled for the 1D EFKM.

Moreover, we present novel results for the singleparticle $c$ - and $f$-electron spectral function of the EFKM. The calculated photoemission spectra are in evidence of a BCS-BEC crossover of the excitonic condensate, trig- 

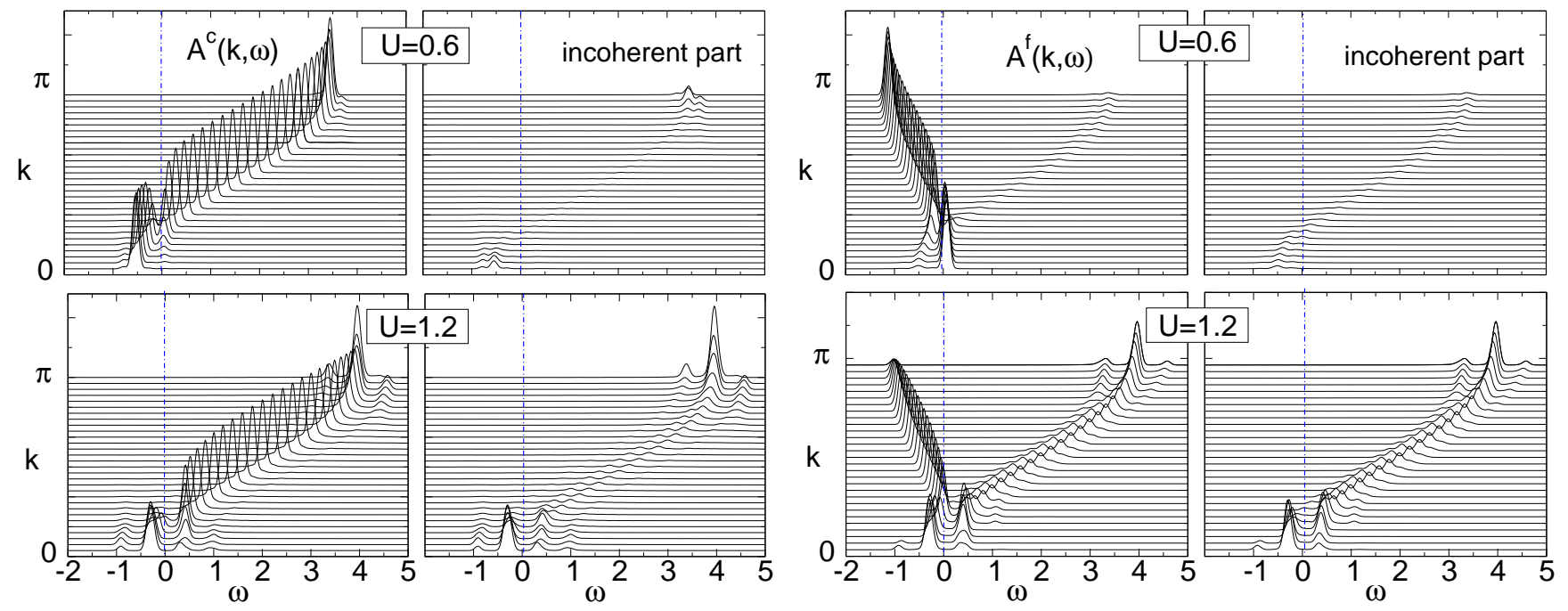

FIG. 7: (Color online) Single-particle spectral functions for $c$ (left-hand panels) and $f$ electrons (right-hand panels) in 1D half-filled EFKM with $\varepsilon^{f}=-1.7, t^{f}=-0.3, T=10^{-3}$. As in Fig. 6] the total spectra are contrasted to the 'incoherent' contributions.

gered by the Coulomb interaction. Thereby the character of the electron-hole pairs changes from the many-body bound state associated with the Cooper-type instability (weak-to-intermediate coupling BCS-side) to the twobody (tightly-bound exciton) bound-state (intermediateto-strong coupling BEC side), where Fermi surface effects are negligible. Hallmark of the BCS-BEC crossover in the quasiparticle spectra is a substantial spectral weight transfer from the coherent to the incoherent part of the spectrum. A more thorough analysis of the pairing fluctuations, which are expected to be strongly enhanced in the BCS-BEC transition region, e.g. by calculating the dynamical pair-susceptibilities within the PRM approach, would be a worthwhile goal of forthcoming studies.

\section{Acknowledgments}

The authors thank F. X. Bronold, B. Bucher, D. Ihle, C. Monney, P. Wachter, and B. Zenker for valuable discussions. This work was supported by the DFG through the research program SFB 652, B5.
1 N. F. Mott, Philos. Mag. 6, 287 (1961).

${ }^{2}$ R. Knox, in Solid State Physics, edited by F. Seitz and D. Turnbull (Academic Press, New York, 1963), p. Suppl. 5 p. 100.

3 D. Jérome, T. M. Rice, and W. Kohn, Phys. Rev. 158, 462 (1967).

4 P. B. Littlewood, P. R. Eastham, J. M. J. Keeling, F. M. Marchetti, B. D. Simons, and M. H. Szymaska, J. Phys.: Condens. Matter 16, 3597 (2004).

${ }^{5}$ L. V. Butov, C. W. Lai, A. L. Ivanov, A. C. Gossard, and D. S. Chemla, Nature 417, 47 (2002).

${ }^{6}$ H. Cercellier, C. Monney, F. Clerc, C. Battaglia, L. Despont, M. G. Garnier, H. Beck, and P. Aebi, Phys. Rev. Lett. 99, 146403 (2007); C. Monney, H. Cercellier, F. Clerc, C. Battaglia, E. F. Schwier, C. Didiot, M. G. Garnier, H. Beck, and P. Aebi, Phys. Rev. B 79, 045116 (2009); C. Monney, E. F. Schwier, C. Battaglia, M. G. Garnier, N. Mariotti, C. Didiot, H. Beck, P. Aebi, H. Cercellier, J. Marcus, et al., preprint, URL arXiv:0911.0327.

7 Y. Wakisaka, T. Sudayama, K. Takubo, T. Mizokawa, M. Arita, H. Namatame, M. Taniguchi, N. Katayama,
M. Nohara, and H. Takagi, Phys. Rev. Lett. 103, 026402 (2009).

8 J. Neuenschwander and P. Wachter, Phys. Rev. B 41, 12693 (1990); B. Bucher, P. Steiner, and P. Wachter, Phys. Rev. Lett. 67, 2717 (1991); P. Wachter, B. Bucher, and J. Malar, Phys. Rev. B 69, 094502 (2004); B. Bucher, T. Park, J. D. Thompson, and P. Wachter (2008), URL arXiv:0802.3354 [cond-mat.str-el]

9 F. X. Bronold and H. Fehske, Phys. Rev. B 74, 165107 (2006).

10 J. des Cloizeaux, J. Chem. Phys. Solids 26, 259 (1965); W. Kohn, in Many Body Physics, edited by C. de Witt and R. Balian (Gordon \& Breach, New York, 1968); A. N. Kozlov and L. A. Maksimov, Sov. Phys. JETP 21, 790 (1965); J. Zittartz, Physical Review 165, 612 (1968).

11 D. Kremp, D. Semkat, and K. Henneberger, Phys. Rev. B 78, 125315 (2008).

12 H. Haug and S. Schmitt-Rink, Prog. Quant. Electr. 9, 3 (1984).

13 F. X. Bronold, H. Fehske, and G. Röpke, J. Phys. Soc. Jpn. Suppl. A 76, 27 (2007); F. X. Bronold and H. Fehske, 

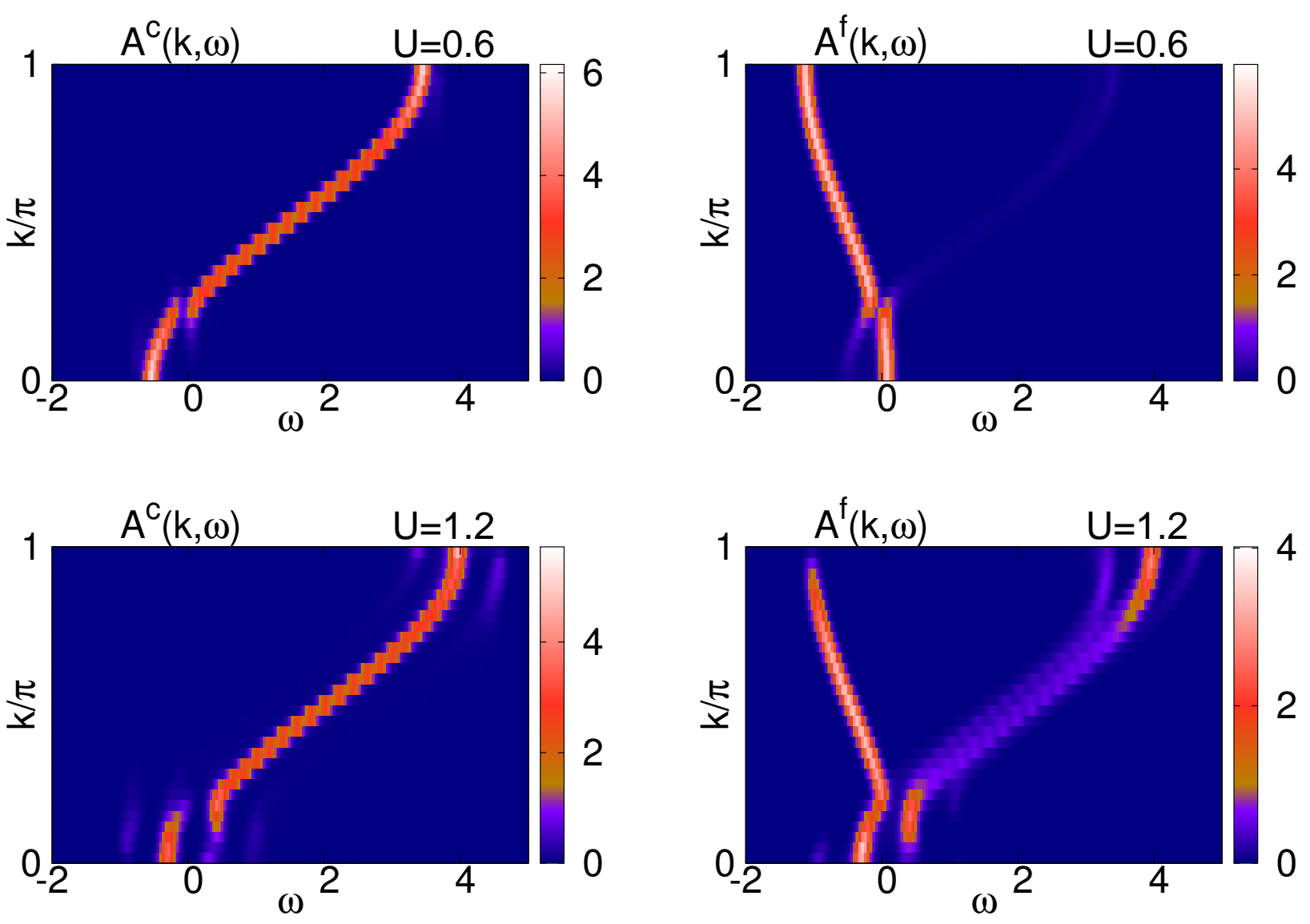

FIG. 8: (Color online) Intensity plots of the $c$ - (left-hand panels) and $f$ - (right-hand panels) electron spectral functions $A^{\eta}(k, \omega)$, presented in Fig. 7 for $\varepsilon^{f}=-1.7, t^{f}=-0.3$ at $U=0.6$ (upper panels) and $U=1.2$ (lower panels).

Superlattices and Microstructures 43, 512 (2008).

${ }^{14}$ C. D. Batista, J. E. Gubernatis, J. Bonča, and H. Q. Lin, Phys. Rev. Lett. 92, 187601 (2004).

15 C. D. Batista, Phys. Rev. Lett. 89, 166403 (2002).

16 P. Farkašovský, Phys. Rev. B 77, 155130 (2008).

17 C. Schneider and G. Czycholl, Eur. Phys. J. B 64, 43 (2008).

18 B. Zenker, D. Ihle, F. X. Bronold, and H. Fehske, Phys. Rev. B 1, 1 (2010).

19 P. M. R. Brydon, Phys. Rev. B 77, 045109 (2008).

20 A. J. Leggett, in Modern Trends in the Theory of Condensed Matter, edited by A. Pekalski and R. Przystawa (Springer-Verlag, Berlin, 1980); C. Comte and P. Nozières, J. Phys. (France) 43, 1069 (1982).

21 D. Ihle, M. Pfafferott, E. Burovski, F. X. Bronold, and H. Fehske, Phys. Rev. B 78, 193103 (2008).

22 J. T. Stewart, J. P. Gaebler, and D. S. Jin, Nature 454, 744 (2008); S. Tsuchiya, R. Watanabe, and Y. Ohashi (2010), preprint, URL arXiv:1002.3016.

23 A. Hübsch and K. W. Becker, Eur. Phys. J. B 52, 345 (2006).

24 A. Hübsch, S. Sykora, and K. W. Becker (2008), preprint, URL arXiv:0809.3360.

25 A. Hübsch and K. W. Becker, Phys. Rev. B 71, 155116
(2005); S. Sykora, A. Hübsch, K. W. Becker, G. Wellein, and H. Fehske, Phys. Rev. B 71, 045112 (2005); K. W. Becker, S. Sykora, and V. Zlatic, Phys. Rev. B 75, 075101 (2007); S. Sykora and K. W. Becker, and H. Fehske, Phys. Rev. B 81, 195127 (2010).

26 S. Sykora and K. W. Becker, Phys. Rev. B 80, 014511 (2009).

27 S. Kehrein, The Flow Equation Approach to Many-Particle Systems, vol. 217 of Springer Tracts in Modern Physics (Springer, Berlin, 2006).

28 E. Körding and F. Wegner, J. Phys. A: Math. Gen. 39, 1231 (2006).

29 K. W. Becker, A. Hübsch, and T. Sommer, Phys. Rev. B 66, 235115 (2002).

30 S. Sykora, A. Hübsch, and K. W. Becker, Europhys. Lett. 85, 57003 (2009).

31 N. V. Phan, Ph.D. thesis, Technical University Dresden (2009).

32 S. Zhang, J. Carlson, and J. E. Gubernatis, Phys. Rev. Lett. 74, 3652 (1994).

33 S. Ejima, F. Gebhard, and S. Nishimoto, Europhys. Lett. 70, 492 (2005); S. Ejima and H. Fehske, Europhys. Lett. 89, 27001 (2009). 